\title{
Toward a refined classification of class I dithiol glutaredoxins from poplar: biochemical basis for the definition of two subclasses
}

\author{
Jérémy Couturier ${ }^{1,2}$, Jean-Pierre Jacquot ${ }^{1,2}$ and Nicolas Rouhier ${ }^{1,2 *}$ \\ 1 Interactions Arbres - Microorganismes, Université de Lorraine, UMR1136, Vandoeuvre-lès-Nancy, France \\ 2 Interactions Arbres - Microorganismes, Institut National de la Recherche Agronomique, UMR1136, Champenoux, France
}

Edited by:

Andreas Meyer, University of Bonn, Germany

Reviewed by:

Mirko Zaffagnini, University of

Bologna, Italy

Christophe Riondet, UMR 5096,

France

*Correspondence:

Nicolas Rouhier, Faculté des

Sciences, Interactions Arbres -

Microorganismes, Université de

Lorraine, UMR1136, BP 239,

54506 Vandoeuvre, France

e-mail: nicolas.rouhier@

univ-lorraine.fr
Glutaredoxins (Grxs) are small oxidoreductases particularly specialized in the reduction of protein-glutathione adducts. Compared to other eukaryotic organisms, higher plants present an increased diversity of Grxs which are organized into four classes. This work presents a thorough comparative analysis of the biochemical and catalytic properties of dithiol class I Grxs from poplar, namely GrxC1, GrxC2, GrxC3, and GrxC4. By evaluating the in vitro oxidoreductase activity of wild type and cysteine mutated variants and by determining their dithiol-disulfide redox potentials, $\mathrm{p} K_{a}$ values of the catalytic cysteine, redox state changes in response to oxidative treatments, two subgroups can be distinguished. In accordance with their probable quite recent duplication, $\mathrm{GrxC} 1$ and $\mathrm{GrxC2}$ are less efficient catalysts for the reduction of dehydroascorbate and hydroxyethyldisulfide compared to $\mathrm{GrxC} 3$ and $\mathrm{GrxC4}$, and they can form covalent dimers owing to the presence of an additional $\mathrm{C}$-terminal cysteine $\left(\mathrm{Cys}_{C}\right)$. Interestingly, the second active site cysteine $\left(\mathrm{Cys}_{\mathrm{B}}\right)$ influences the reactivity of the catalytic cysteine $\left(\mathrm{Cys}_{\mathrm{A}}\right)$ in $\mathrm{GrxC} 1$ and $\mathrm{GrxC2}$ as already observed with GrxC5 (restricted to $A$. thaliana), but not in $\mathrm{GrxC3}$ and $\mathrm{C} 4$. However, all proteins can form an intramolecular disulfide between the two active site cysteines $\left(\mathrm{Cys}_{\mathrm{A}}-\mathrm{Cy} \mathrm{s}_{\mathrm{B}}\right)$ which could represent either a protective mechanism considering that this second cysteine is dispensable for deglutathionylation reaction or a true catalytic intermediate occurring during the reduction of particular disulfide substrates or in specific conditions or compartments where glutathione levels are insufficient to support Grx regeneration. Overall, in addition to their different sub-cellular localization and expression pattern, the duplication and maintenance along evolution of several class I Grxs in higher plants can be explained by the existence of differential biochemical and catalytic properties.

Keywords: cysteine oxidation, deglutathionylation, disulfide bond, glutaredoxin, redox potential

\section{INTRODUCTION}

Glutaredoxins (Grxs) are oxidoreductases sharing a conserved 3D structure with members of the thioredoxin (Trx) superfamily. They are present in most living organisms except in some bacterial and archaeal phyla (Rouhier et al., 2008; Alves et al., 2009). The primary function of Grxs was long thought to be the reduction of disulfide bonds and more particularly those formed between reduced glutathione (GSH) and a protein cysteinyl residue, a process known as glutathionylation, but as explained below, specific Grx members could rather serve as iron-sulfur (FeS) cluster transfer proteins (Rouhier, 2010). Glutathionylation is a post-translational modification which potentially fulfills several functions. It can constitute an intermediate of some reaction mechanisms, but it can also modulate protein function and serve as a signaling mechanism or protect cysteine residues from irreversible oxidation in plants (Zaffagnini et al., 2012b) and in animals (Dalle-Donne et al., 2009). Although a few additional proteins, as some specific thioredoxins, can catalyze deglutathionylation reactions, Grxs are likely the major deglutathionylation system, at least in plants (Bedhomme et al., 2012; Chibani et al., 2012). They are usually regenerated via a NADPH/glutathione reductase (GR)/GSH system, but a few Grxs can be reduced by ferredoxin- or NADPH-dependent thioredoxin reductases (Johansson et al., 2004; Zaffagnini et al., 2008). Depending on the Grx isoforms and target proteins or substrates considered, Grxs can use different catalytic mechanisms, called monothiol and dithiol mechanisms (Rouhier et al., 2008). The monothiol mechanism is used for the reduction of glutathionylated proteins and requires a priori only the catalytic cysteine (the first or more $\mathrm{N}$-terminal of the two active site cysteines, referred to as $\left.\mathrm{Cys}_{\mathrm{A}}\right)$. It performs a nucleophilic attack on the proteinglutathione adduct, the Grx becoming glutathionylated. This oxidized Grx is regenerated by reduction via a GSH molecule. The dithiol mechanism requires the catalytic cysteine but also a recycling cysteine which could be either the second active site cysteine $\left(\mathrm{Cys}_{B}\right)$ or an additional extra active site cysteine $\left(\mathrm{Cys}_{C}\right)$. If the target protein is glutathionylated, the first step is similar to the monothiol mechanism, but the glutathione-adduct formed on 
Grx is solved by one of these recycling cysteines instead of GSH. The resulting disulfide is then either reduced by two molecules of GSH or by a thioredoxin reductase. If the target protein has an intra or inter-molecular disulfide, the mechanism is comparable to the one used by Trxs. The catalytic cysteine forms a transient mixed disulfide with one of the cysteines of the target protein, which is then resolved by the recycling cysteine. As above, final regeneration occurs via two molecules of GSH or via a thioredoxin reductase.

Based on the active site sequence, Grxs were initially categorized into two classes, a dithiol $(\mathrm{CP}[\mathrm{Y} / \mathrm{F}] \mathrm{C}$ motif) and a monothiol (CGFS motif) class (Rodriguez-Manzaneque et al., 1999) that were subsequently renamed classes I and II, respectively (Couturier et al., 2009a). Analyses of Grx distribution and evolution in archaea, bacteria, and eukaryotes suggested that Grx domains of classes I and II have evolved through duplication and divergence from one initial gene present in the last common ancestor of all organisms (Alves et al., 2009). Two additional classes are found in land plants (Couturier et al., 2009a). The Grx isoforms belonging to class III are specific to land plants and those from class IV are also present in few algae and animals. There is no biochemical or functional information on the latter Grxs yet. From genetic analyses, it appears that class III Grx isoforms also named CC-type Grxs or ROXY play a role in plant development and in pathogen defense mechanisms, reviewed in (Meyer et al., 2012). These functions are related to their ability to interact with TGA transcription factors, likely regulating their redox states. However, the biochemical properties of these Grxs have not been studied in detail yet, because of the difficulty to produce soluble recombinant proteins (Couturier et al., 2010). Only one study demonstrated that Arabidopsis thaliana GrxS10 is able to regenerate in vitro the mitochondrial type IIF peroxiredoxin (Finkemeier et al., 2005). The Grxs from class II are particular considering their involvement in the maturation of Fe-S clusters and in the regulation of iron homeostasis (Rouhier et al., 2010), which is likely related to their capacity to bind labile Fe$S$ clusters and to transfer them to target proteins (Bandyopadhyay et al., 2008). On the other hand, though many studies performed with class II Grxs from various sources indicated that they have no or very poor efficiency for the reduction of the traditionally tested glutathionylated substrates using a GR/GSH regeneration system, at least one study indicated that they have a reductase activity. Indeed, Chlamydomonas reinhardtii Grx3 is able to reduce in vitro a glutathionylated $\mathrm{A}_{4}$-glyceraldehyde-3-phosphate dehydrogenase (Zaffagnini et al., 2008). Interestingly, it can be regenerated by the ferredoxin-thioredoxin reductase but not by the GSH/GR system. The existence of alternative regeneration systems may explain why the characterization of such activities for class II Grxs has been retarded. Overall, the demonstrated capacity of several class I Grxs to provide electrons to a battery of enzymes, as peroxiredoxins and methionine sulfoxide reductases, enzymes that go through a catalytic cycle which involves glutathionylation of their catalytic cysteine (Rouhier et al., 2001; Vieira Dos Santos et al., 2007; Tarrago et al., 2009), suggests that they are the central and favorite deglutathionylating agents in cells.

In plants, class I regroups six different glutaredoxin members called $\mathrm{GrxC} 1, \mathrm{C} 2, \mathrm{C} 3, \mathrm{C} 4, \mathrm{~S} 12$, and $\mathrm{C} 5$, the latter being restricted to Brassicaceae (Couturier et al., 2011). The cytoplasmic GrxC1 and the plastidial GrxC5 are able to bind an $\left[\mathrm{Fe}_{2} \mathrm{~S}_{2}\right]$ cluster, at least in vitro into recombinant proteins (Rouhier et al., 2007; Couturier et al., 2011). The biochemical and structural characterization of the two plastidial isoforms, GrxS12 and GrxC5, confirmed that the apoforms of these Grxs reduce glutathionylated proteins with catalytic properties quite similar to other plant and non-plant class I Grxs (Couturier et al., 2009b, 2011). Moreover, poplar GrxS12 was shown to be sensitive and temporarily inactivated after treatments with oxidizing compounds such as hydrogen peroxide $\left(\mathrm{H}_{2} \mathrm{O}_{2}\right)$, nitrosoglutathione (GSNO), and oxidized glutathione (GSSG) (Zaffagnini et al., 2012a). Considering the very electronegative redox potential of the glutathione adduct formed on the catalytic Cys $(-315 \mathrm{mV}$ at $\mathrm{pH} 7.0)$, the reduction of GrxS12 necessitates a quite low redox potential of the GSH/GSSG couple in the chloroplast. Thus, GrxS12 may act as a stress-related redox regulator allowing glutathione to play a signaling role in some oxidizing conditions by maintaining the glutathionylation of its target proteins over the duration of the stress period. Several class I Grxs, C. reinhardtii Grx1 and Grx2 (Gao et al., 2010), A. thaliana GrxC1, C2 (Riondet et al., 2012) and GrxC5 (Couturier et al., 2011) and poplar GrxC1, GrxC4, and GrxS12 (Rouhier et al., 2002, 2007; Couturier et al., 2009b; Zaffagnini et al., 2012a) have been partially or extensively characterized at the biochemical level, but some members have not yet been studied and there is no thorough and comparative study allowing to understand if the existence of several members is only related to their sub-cellular localization or to their expression pattern or whether some biochemical properties can partially explain their diversity.

From the biochemical analyses performed here with the four dithiol class I Grxs existing in poplar, GrxC1 to GrxC4 (the fifth member, GrxS12, has a monothiol active site and was characterized previously), two sub-groups with distinct properties emerged. In addition, this work provides new insights on the existence of different oxidation forms involving the active site cysteines $\left(\mathrm{Cys}_{\mathrm{A}}\right.$ and $\left.\mathrm{Cys}_{\mathrm{B}}\right)$ or an additional $\mathrm{C}$-terminal cysteine $\left(\mathrm{Cys}_{C}\right)$ and on their potential role for the reaction mechanism.

\section{MATERIALS AND METHODS SITE-DIRECTED MUTAGENESIS}

The construction of pET3d expression plasmids containing poplar GrxC1, C2, C3, and C4 was described previously (Rouhier et al., 2002, 2007). All cysteine residues of GrxC2, C3 were individually substituted into serines using two complementary mutagenic primers (listed in Table S1). The corresponding mutated proteins are called GrxC2 C23S, C26S, or C80S, GrxC3 C37S or C40S. The recombinant proteins GrxC1 C31S, C34S, C88S, and GrxC4 C27S and C30S were prepared previously (Rouhier et al., 2002, 2007). Two additional mutants, in which only the catalytic cysteine is remaining, GrxC1 C34/88S and GrxC2 C26/80S, have also been cloned.

\section{HETEROLOGOUS EXPRESSION AND PURIFICATION OF RECOMBINANT PROTEINS IN E. coli}

For protein expression, cultures of the E. coli BL21(DE3) strain, containing the pSBET plasmid and co-transformed with the 
different recombinant $\mathrm{pET} 3 \mathrm{~d}$ plasmids, were successively amplified up to $2.4 \mathrm{~L}$ in $\mathrm{LB}$ medium at $37^{\circ} \mathrm{C}$ supplemented with $50 \mu \mathrm{g} / \mathrm{mL}$ of ampicillin and kanamycin. Induction of protein expression was performed at exponential phase by adding $100 \mu \mathrm{M}$ isopropyl $\beta$-D-thiogalactopyranoside for $4 \mathrm{~h}$ at $37^{\circ} \mathrm{C}$. After centrifugation ( $20 \mathrm{~min}$ at $4400 \mathrm{~g}$ ), the cell pellets were resuspended in about $20 \mathrm{~mL}$ of $\mathrm{TE} \mathrm{NaCl}$ buffer $(30 \mathrm{mM}$ Tris- $\mathrm{HCl}$ pH 8.0, $1 \mathrm{mM}$ EDTA, $200 \mathrm{mM} \mathrm{NaCl}$ ) and eventually conserved at $-20^{\circ} \mathrm{C}$. Cell lysis was performed by sonication and the soluble and insoluble fractions were separated by centrifugation for $45 \mathrm{~min}$ at $27,000 \mathrm{~g}$.

Purification of proteins was carried out in three steps. The soluble fraction was first precipitated by ammonium sulfate from 0 to $40 \%$ and then to $80 \%$ of the saturation. The $40-80 \%$ ammonium sulfate-precipitated fraction was subjected to gel filtration chromatography (ACA44 gel) equilibrated with TE $\mathrm{NaCl}$ buffer. After dialysis against TE (30 mM Tris- $\mathrm{HCl}$ pH 8.0, 1 mM EDTA) buffer and concentration, the interesting fractions were loaded to a DEAE (diethylaminoethyl) sepharose column equilibrated in TE buffer. GrxC1 WT, GrxC2 WT and their corresponding cysteinic mutants passed through the column, whereas GrxC3 WT, GrxC4 WT and their respective cysteinic mutants were retained and eluted using a linear $0-0.4 \mathrm{M} \mathrm{NaCl}$ gradient. The purest fractions, as judged by SDS-PAGE analysis, were pooled and dialyzed against TE buffer by ultrafiltration in Amicon cells equipped with a YM10 membrane. Finally, the fractions were concentrated and stored at $-20^{\circ} \mathrm{C}$ until further use. Protein purity was checked by SDS-PAGE, and protein concentrations were determined spectrophotometrically using the corresponding molar extinction coefficients at $280 \mathrm{~nm}$ of $10,095 \mathrm{M}^{-1} \mathrm{~cm}^{-1}$ for GrxC1 WT and its monocysteinic mutants, $9970 \mathrm{M}^{-1} \mathrm{~cm}^{-1}$ for GrxC1 C34/88S, $8605 \mathrm{M}^{-1} \mathrm{~cm}^{-1}$ for GrxC2 WT and its monocysteinic mutants, $8480 \mathrm{M}^{-1} \mathrm{~cm}^{-1}$ for GrxC2 C26/80S, $7575 \mathrm{M}^{-1} \mathrm{~cm}^{-1}$ for GrxC3 WT, $7450 \mathrm{M}^{-1} \mathrm{~cm}^{-1}$ for GrxC3 C37S and C40S, $4595 \mathrm{M}^{-1} \mathrm{~cm}^{-1}$ for GrxC4 WT and $4470 \mathrm{M}^{-1} \mathrm{~cm}^{-1}$ for GrxC4 C27S and C30S.

\section{ENZYMATIC ACTIVITIES}

The thioltransferase activities [2-hydroxyethyl-disulfide (HED) and dehydroascorbate (DHA) assays] were measured as described previously (Couturier et al., 2011) using as purified non-reduced proteins. Briefly, measurements were performed at $25^{\circ} \mathrm{C}$ in steady-state conditions by following NADPH oxidation at $340 \mathrm{~nm}$ in the presence of a Grx reducing system which is composed of NADPH, GR, and GSH. The reaction was started by adding Grx after a 3-min pre-incubation time and Grx activity was corrected by subtracting the spontaneous reduction rate observed in the absence of Grx. Because of the spontaneous reaction of GSH with the substrates, we can generally not work at saturating concentrations of all substrates using these assays. Enzyme and substrate concentrations used are indicated in the legend of figures and tables. The activity was expressed as nmol of NADPH oxidized/nmol of Grx/s using a molar extinction coefficient of $6220 \mathrm{M}^{-1} \mathrm{~cm}^{-1}$ at $340 \mathrm{~nm}$ for NADPH. Three independent experiments were performed at each substrate concentration, and $k_{\text {cat }}$ and apparent $K_{m}$ values were calculated by non-linear regression using the Michaelis-Menten equation using the program GraphPad Prism 4.

\section{PREPARATION AND ANALYSIS OF OXIDIZED GrXs}

Around $10 \mathrm{mg}$ of protein was reduced using $40 \mathrm{mM}$ DTT in $500 \mu \mathrm{l}$ of $30 \mathrm{mM}$ Tris- $\mathrm{HCl} \mathrm{pH} 8.0$ buffer for $2 \mathrm{~h}$ at $25^{\circ} \mathrm{C}$ followed by desalting on G25 column pre-equilibrated with $30 \mathrm{mM}$ Tris$\mathrm{HCl} \mathrm{pH} 8.0$ buffer. Oxidized proteins were prepared by incubating $500 \mu \mathrm{M}$ reduced poplar Grxs in ca $200 \mu \mathrm{L}$ with a 10 fold excess GSSG or $\mathrm{H}_{2} \mathrm{O}_{2}$ or a 5 fold excess GSNO for $2 \mathrm{~h}$ at $25^{\circ} \mathrm{C}$ before desalting on G25 columns. The protein oxidation state was then analyzed by electrospray ionization mass spectrometry analysis as described previously (Couturier et al., 2011).

For alkylation assays, reduced Grxs treated or not with oxidants were diluted to $10 \mu \mathrm{M}$ into $50 \mu \mathrm{l}$ of $30 \mathrm{mM}$ Tris- $\mathrm{HCl} \mathrm{pH}$ 8.0 buffer and precipitated on ice for $30 \mathrm{~min}$ with one volume of $20 \%$ trichloroacetic acid (TCA). After centrifugation (10 min at $13,000 \mathrm{~g}$ ) and washing with $100 \%$ acetone, the pellet was resuspended into $10 \mu \mathrm{l}$ of $100 \mathrm{mM}$ Tris- $\mathrm{HCl} \mathrm{pH} 8.0,1 \%$ SDS containing $2 \mathrm{mM}$ of methoxyl-PEG maleimide of $2 \mathrm{kDa}$ (mPEG maleimide) which alkylates free thiol groups. The protein mixture was then separated on non-reducing 15\% SDS-PAGE.

For midpoint redox potential titrations, $\mathrm{Cys}_{\mathrm{A}}$ glutathionylated forms were obtained by the above described GSNO treatment of the double cysteinic mutants (GrxC1 C34/88S, GrxC2 C26/80S) and of the monocysteinic mutants (GrxC3 C40S and GrxC4 $\mathrm{C} 30 \mathrm{~S})$. The intramolecular disulfide bond $\left(\mathrm{Cys}_{\mathrm{A}}-\mathrm{Cys} \mathrm{B}_{\mathrm{B}}\right)$ containing proteins were obtained by the above described $\mathrm{H}_{2} \mathrm{O}_{2}$ treatment but using GrxC1 C88S, GrxC2 C80S and GrxC3 and GrxC4 WT isoforms.

\section{p $K_{a}$ DETERMINATION OF THE CATALYTIC CYSTEINE}

The $\mathrm{p} K_{a}$ measurements of N-terminal active site cysteines $\left(\mathrm{Cys}_{\mathrm{A}}\right)$ have been performed with WT Grx isoforms following a procedure described in (Gallogly et al., 2008). Briefly, $3 \mu \mathrm{M}$ reduced Grx was incubated with or without $300 \mu \mathrm{M}$ iodoacetamide (IAM) in $100 \mathrm{mM}$ sodium citrate or phosphate buffers ranging from $\mathrm{pH}$ 2.0 to 7.0. Following this pre-incubation step, Grx activity was determined by adding an aliquot of the pre-incubation mixture to the HED assay described above. The percentages of remaining activity at each $\mathrm{pH}$ were determined by comparing the activity of the enzyme incubated with and without IAM and an adaptation of the Henderson-Hasselbach equation (Gallogly et al., 2008) was used for $\mathrm{p} K_{a}$ value determination using the program GraphPad Prism 4.

\section{MIDPOINT REDOX POTENTIAL $\left(E_{m}\right)$ DETERMINATION}

Oxidation-reduction titrations using the fluorescence of the adduct formed between protein free thiols and monobromobimane $(\mathrm{mBBr})$ were carried out at ambient temperature in $200 \mu \mathrm{l}$ of $100 \mathrm{mM}$ HEPES pH 7.0 buffer containing $10 \mu \mathrm{M}$ of oxidized proteins, either glutathionylated or with an intramolecular disulfide-bond, and defined mixtures of oxidized and reduced DTT or glutathione for more positive values to set the ambient potential $\left(E_{h}\right)$. Total concentration of DTT or glutathione was $2 \mathrm{mM}$. After $2 \mathrm{~h}$ incubation, $\mathrm{mBBr}$ was added at a final concentration of $2 \mathrm{mM}$ and the reaction was carried out in the dark for $20 \mathrm{~min}$. The reaction mixture was then precipitated on ice for $30 \mathrm{~min}$ with one volume of $20 \%$ TCA. After centrifugation 
(10 $\mathrm{min}$ at $13,000 \mathrm{~g}$ ) and washing with $100 \%$ acetone, the pel-

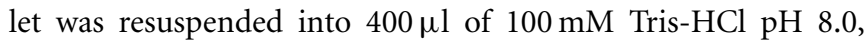
$1 \%$ SDS. Fluorescence emission of the resulting solution was measured at $472 \mathrm{~nm}$ after excitation at $380 \mathrm{~nm}$ using a variant Cary Eclipse (Agilent). Values were transformed into percentages of reduced protein and fitted to the Nernst equation using non-linear regression for $E_{m}$ value determination.

\section{RESULTS \\ KINETIC COMPARISON OF POPLAR CLASS I Grxs}

An amino acid sequence alignment of the predicted mature forms of all poplar class I Grxs reveals several conserved residues, particularly those around the active sites and those involved in glutathione binding (Figure 1). However, GrxC1 and GrxC2 present CGYC and CPFC active site motifs, respectively, different from the CPYC active site found in GrxC3 and GrxC4. Interestingly, contrary to GrxC3 and GrxC4, GrxC1 and GrxC2 isoforms possess an additional cysteine $\left(\mathrm{Cys}_{C}\right)$ located in their C-terminal part in a IGGCD motif, as in human Grx1, E. coli Grx3 and the two other class I Grxs found in plants, GrxC5 and GrxS12 (Figure 1).
With the aim of comparing the redox and kinetic properties of all dithiol Grxs (GrxC5 is not found in poplar) and understanding why these duplicated genes have been retained during evolution, we have performed a thorough biochemical analysis of the corresponding recombinant proteins.

The catalytic properties of each isoform were first determined by measuring their reductase activity using the classical HED and DHA assays, which measure the reduction of glutathionylated$\beta$-mercaptoethanol or of dehydroascorbate, respectively. All proteins display a better catalytic efficiency $\left(k_{c a t} / K_{m}\right)$ in the HED test with a difference of two orders of magnitude (Table 1). In both assays, the kinetic parameters determined for each protein follow the same tendency. GrxC3 and C4 have quite comparable turnover numbers, with $k_{\text {cat }}$ values about 10 fold higher than those of $\mathrm{GrxC} 1$ and $\mathrm{C} 2$. In terms of catalytic efficiencies, the dichotomy between GrxC1/C2 and GrxC3/C4 is clear but it is often below this factor of 10 because GrxC3 and $\mathrm{C} 4$ have higher apparent $K_{m}$ values for both substrates. Overall, GrxC1 always exhibited the lowest catalytic efficiency and GrxC4 the highest. Concerning the apparent affinity for GSH, although there

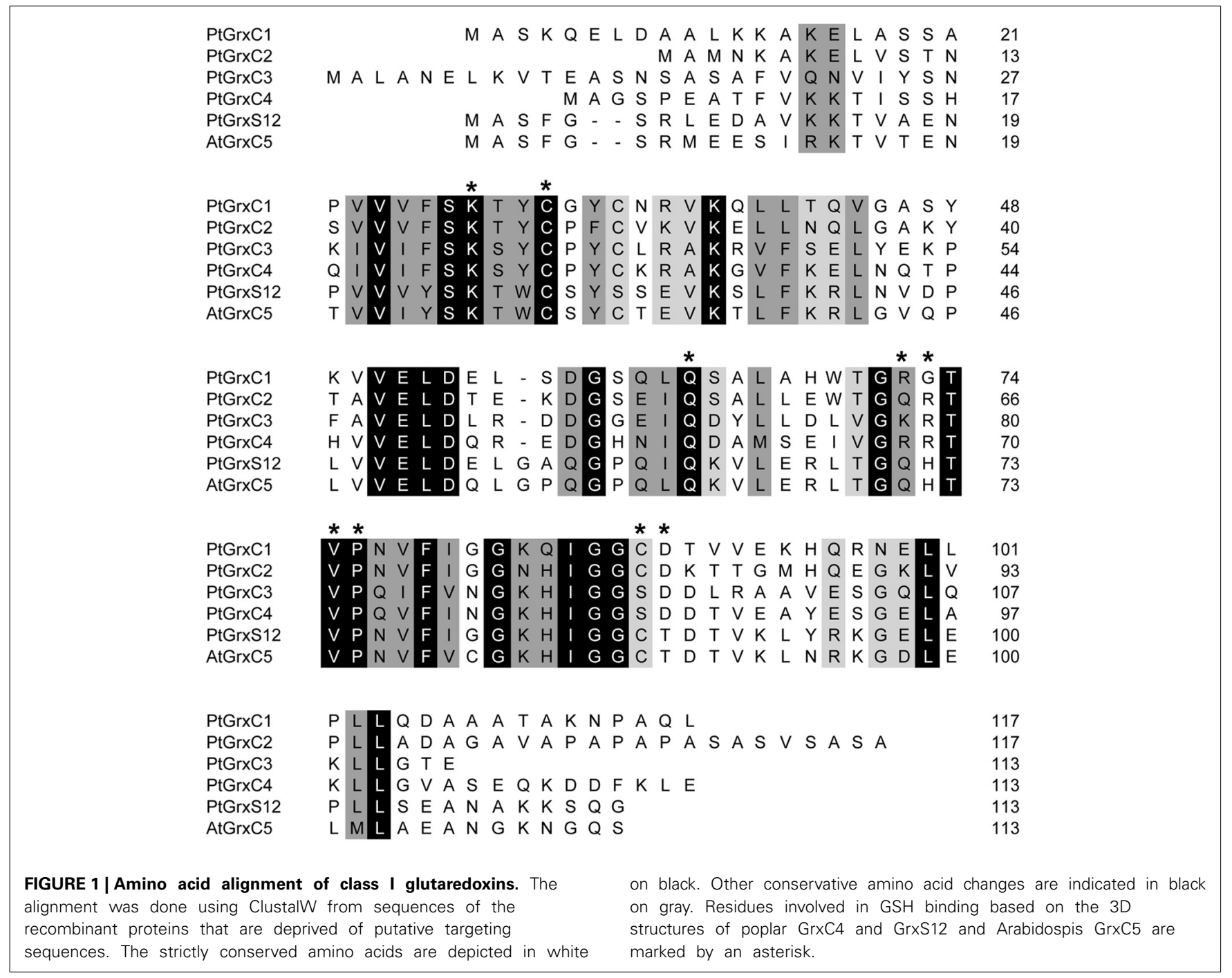


Table 1 | Kinetic parameters of poplar dithiol class I glutaredoxins.

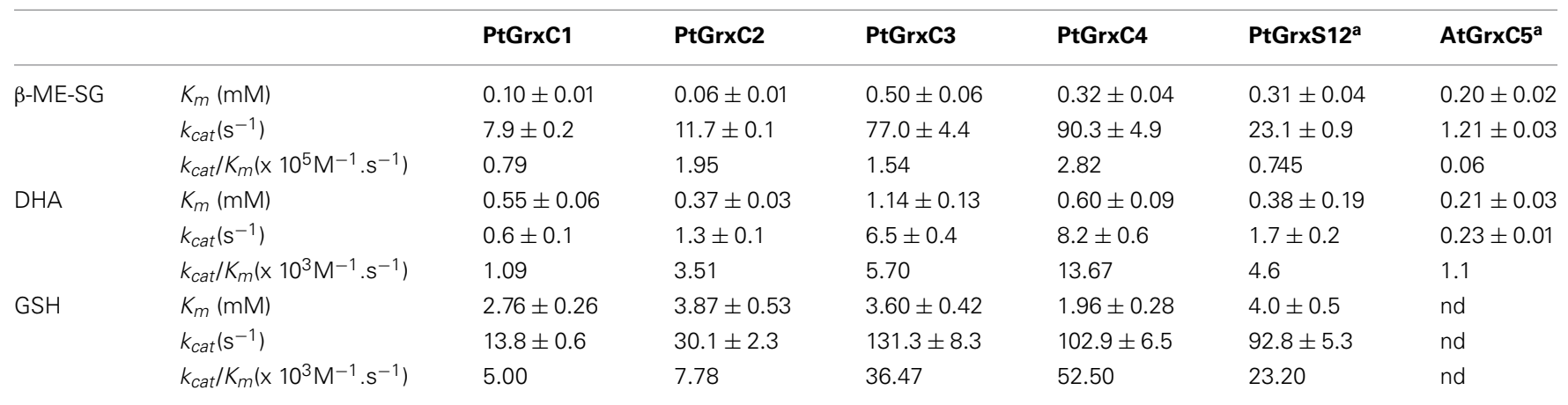

The apparent $K_{m}$ values for $\beta-M E-S G$ (glutathionylated $\beta$-mercaptoethanol) were determined using a HED concentration range of $0.1-1 \mathrm{mM}$ in presence of $2 \mathrm{mM}$ GSH and $100 \mathrm{nM}$ enzyme for GrxC1, $50 \mathrm{nM}$ for GrxC2, $10 \mathrm{nM}$ for GrxC3 and GrxC4. The apparent $K_{m}$ values for DHA were determined using a DHA concentration range of 0.1-1 mM in presence of $2 \mathrm{mM} \mathrm{GSH}$ and $2 \mu \mathrm{M}$ enzyme for GrxC1, $1 \mu \mathrm{M}$ for GrxC2, $200 \mathrm{nM}$ for GrxC3, and $150 \mathrm{nM}$ for GrxC4. The apparent $K_{m}$ values for GSH were determined using a GSH concentration range of $0.25-5 \mathrm{mM}$ in the presence of $0.7 \mathrm{mM}$ HED and $100 \mathrm{nM}$ enzyme for GrxC1, 50 nM for GrxC2, $10 \mathrm{nM}$ for GrxC3 and GrxC4. The $k_{\mathrm{cat}}$ and $K_{m}$ values were calculated by non-linear regression using the Michaelis-Menten equation. Values are the means $\pm S D$ of three replicates. ${ }^{a}$ Data extracted from the following references Couturier et al. (2009b, 2011), Zaffagnini et al. (2012a). nd, not determined.

are differences in the $K_{m}$ values for GSH between the four isoforms, ranging from $\sim 2.0 \mathrm{mM}$ for GrxC4 to $\sim 3.9 \mathrm{mM}$ for GrxC2, the GrxC1/C2 and GrxC3/C4 dichotomy is not visible using this parameter.

Then, the involvement for the catalytic mechanism of the different cysteine residues present in each isoform was investigated by comparing the activity of WT proteins to their corresponding cysteinic mutants using similar assays at fixed substrate concentrations (Figure 2). As expected, all monocysteinic mutants for the catalytic cysteine Cys (GrxC1 C31S, GrxC2 C23S, GrxC3 $\mathrm{C} 37 \mathrm{~S}$, and GrxC4 C27S) were inactive, confirming that it is indispensable for the reductase activity. On the contrary, mutating Cys $_{C}$, in positions 88 and 80 in GrxC1 and GrxC2, respectively, did not affect catalytic activity. Finally, the mutation of $\mathrm{Cys}_{\mathrm{B}}$ has a different impact depending on the isoforms. The protein variants for this cysteine in GrxC1 and C2 (GrxC1 C34S and GrxC2 C26S) were 5 fold more efficient and 2-3 fold more efficient than the corresponding WT proteins in the HED and DHA assays, respectively. On the other hand, the mutation of this cysteine in GrxC3 and $\mathrm{GrxC} 4$ has almost no impact, since, only a slight decrease was observed in the HED assay for the GrxC3 C40S variant.

\section{OXIDATIVE MODIFICATIONS OF CYSTEINE RESIDUES}

Several observations prompted us to analyze the sensitivity of all dithiol class I Grxs to oxidizing treatments and the resulting changes in their redox state. First, the mutation of $\mathrm{Cys}_{\mathrm{B}}$ increased the catalytic efficiency of GrxC1 and C2 but not of GrxC3 and C4 suggesting possible difference in the reaction mechanisms. Second, although $\mathrm{Cys}_{C}$ does not influence their catalytic activity, the migration of the purified proteins in non-reducing SDS PAGE gels indicated the formation of covalent linkages for GrxC1 and $\mathrm{C} 2$ that are not visible for GrxC3 and C4 (data not shown).

For this purpose, reduced proteins have been treated for $2 \mathrm{~h}$ with a 10 fold excess of GSSG or $\mathrm{H}_{2} \mathrm{O}_{2}$, or a 5 fold excess of GSNO. The resulting redox state was analyzed by mass spectrometry and by protein migration on non-reducing SDS PAGE with or without thiol alkylation by $2 \mathrm{kDa}$ mPEG maleimide, which allows distinguishing reduced and oxidized forms of Grxs. Since reduced mPEG-alkylated proteins and covalent dimers of GrxC1 and GrxC2 migrate roughly at the same position, controls without alkylation have been included (Figure 3). With non-alkylated proteins, we confirmed that an $\mathrm{H}_{2} \mathrm{O}_{2}$ treatment, but not the GSSG and GSNO treatments, promoted the formation of covalent dimers of GrxC1 and GrxC2 (Figures 3A,B) but not of GrxC3 and GrxC4 (Figures 3C,D). Only a small part of GrxC2 dimer is visible after these $2 \mathrm{~h}$ GSSG and GSNO treatments. From the procedure that included an alkylation step, we can further conclude that all treatments led essentially to a complete oxidation of all thiol groups present on all Grxs since almost no shift of migration was observed. A minor form with one free thiol group is visible for GrxC2 after a GSSG treatment. It is even more discrete upon GSNO treatment. For GrxC3 and C4, these oxidized monomeric forms are interpreted as containing intramolecular disulfides between active site cysteines $\left(\mathrm{Cys}_{\mathrm{A}}{ }^{-}\right.$ Cys $_{\mathrm{B}}$ ) (Figures 3C,D). For GrxC1 and C2, these two cysteines should also form this intramolecular disulfide, and $\mathrm{Cys}_{C}$ is either modified by a glutathione or a nitroso (in the case of GSNO) adduct as confirmed by mass spectrometry analyses (see below) or it is involved in an intermolecular disulfide upon $\mathrm{H}_{2} \mathrm{O}_{2}$ treatment. From similar experiments performed with the GrxC1 C88S and GrxC2 C80S mutants (Figures 3E,F), the absence of covalent dimers indicated that $\mathrm{Cys}_{C}$ is indeed involved in dimerization. Moreover, in the context of these variants, after GSSG and GSNO treatments, the proteins mainly contain an active site disulfide bond whereas a small part of the proteins has one remaining free thiol, likely corresponding to glutathionylated intermediates.

In order to identify the type of protein modification formed by these oxidative treatments, all proteins have been analyzed by mass spectrometry (Table 2 and Figures S1-S6). First, all reduced proteins gave a single species with a mass decrease of $c a 131 \mathrm{Da}$ compared to the calculated theoretical molecular masses. It does undoubtedly correspond to the cleavage of the first methionine as expected from the presence of an alanine in second position. The results obtained with GrxC3 and GrxC4 are more easily 


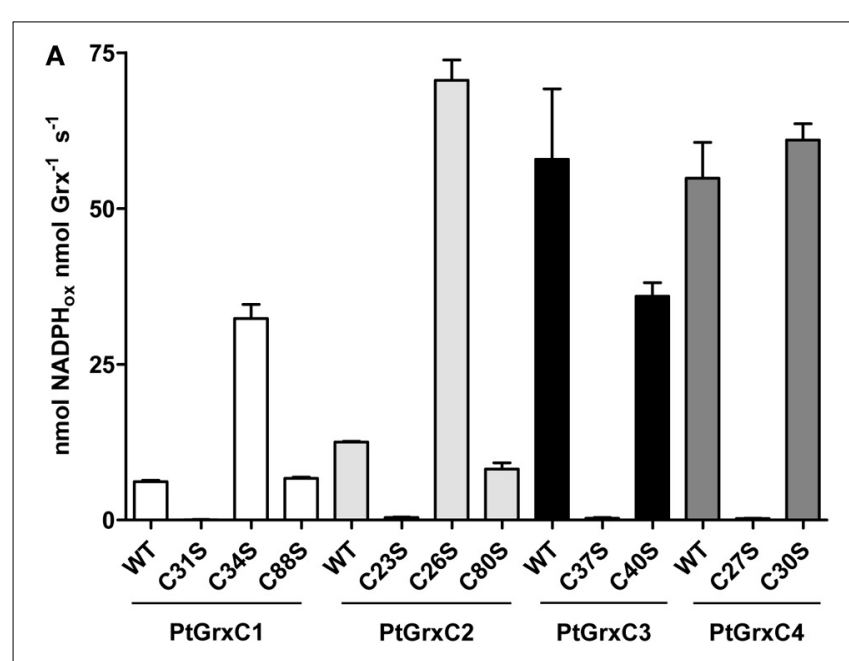

8

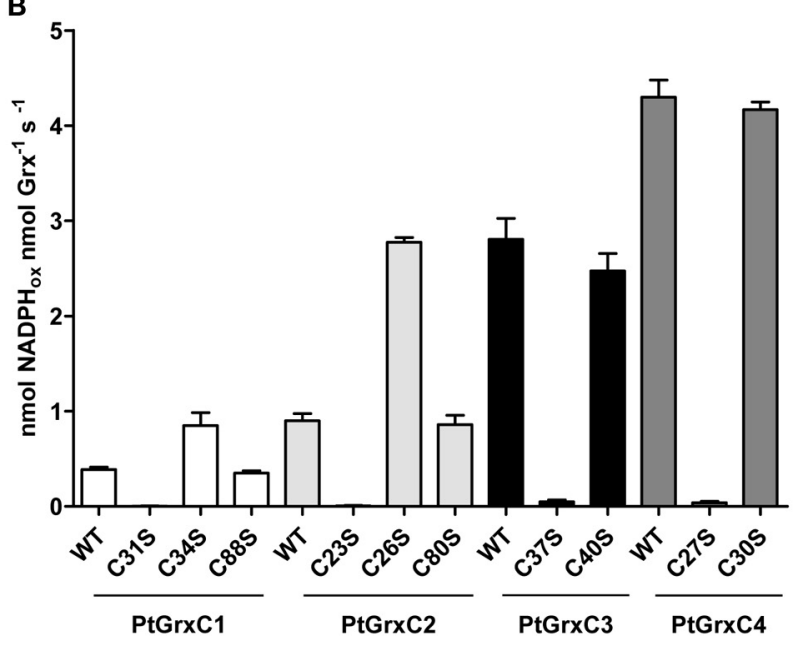

FIGURE 2 | Reductase activity of GrxC1, C2, C3, and C4 and of cysteine-to-serine variants. (A) HED reduction was measured in the presence of $2 \mathrm{mM} \mathrm{GSH}$ and $0.7 \mathrm{mM} \mathrm{HED}$ using either $100 \mathrm{nM} \mathrm{GrxC1}$, 20 nM for GrxC1 C34S, 50 nM GrxC2, 10 nM for GrxC2 C26S, 10 nM GrxC3, or $10 \mathrm{nM} \mathrm{GrxC4}$. (B) DHA reductase activity was measured in the presence of $2 \mathrm{mM} \mathrm{GSH}$ and $1 \mathrm{mM}$ DHA using either $2 \mu \mathrm{M} \mathrm{GrxC1}, 1 \mu \mathrm{M}$ for GrxC1 C34S, $1 \mu$ M GrxC2, 250 nM GrxC2 C26S, 200 nM GrxC3, or 150 nM GrxC4. The data are expressed as nmol NADPH oxidized per nmol enzymes per $\mathrm{s}$ and represent means \pm SD of three separate experiments.

interpreted since these proteins contain only two cysteines. In the presence of $\mathrm{H}_{2} \mathrm{O}_{2}$, and in accordance with the absence of detected free thiols in the previous experiments (Figures 3C,D), GrxC3 and GrxC4 are exclusively detected as a monomeric oxidized form with a decrease of $\sim 2 \mathrm{Da}$ confirming the formation of a $\mathrm{Cys}_{\mathrm{A}}-\mathrm{Cys}_{\mathrm{B}}$ intramolecular disulfide bond. The same forms were primarily obtained upon GSSG and GSNO treatments but a minor monomeric form with a mass increment of 305 Da corresponding to one glutathione adduct was also detected (Table 2). It was not detected on gels presumably because of the presence of minute amounts. Nevertheless, this suggests that the glutathionylated form represents an intermediate for the formation of the intramolecular disulfide.
The results obtained for GrxC1 and GrxC2 are slightly more complex. In response to an $\mathrm{H}_{2} \mathrm{O}_{2}$ treatment, two different species are formed for both proteins. In accordance with the results from alkylation experiments, a major form corresponds to a disulfidebridged dimer having also an intramolecular $\mathrm{Cys}_{\mathrm{A}}-\mathrm{Cys}_{\mathrm{B}}$ disulfide bond. The detected monomeric forms, which have no free thiols or a very small fraction as observed for GrxC2, exhibited a mass increment of 29.5 Da for GrxC1 and 32.2 Da for GrxC2, suggesting the presence of two oxygen atoms. Considering that this overoxidation is not observed for GrxC1 C88S and GrxC2 C80S variants which only form monomeric proteins with an intramolecular disulfide (as indicated by the mass decrease of $2 \mathrm{Da}$ ), the results obtained for the WT proteins are best interpreted as the formation of an intramolecular disulfide between the active site cysteines $\left(\mathrm{Cys}_{\mathrm{A}}-\mathrm{Cys} \mathrm{s}_{\mathrm{B}}\right)$ and of a sulfinic acid on Cys $_{C}$. In the presence of GSSG or GSNO, GrxC1 and GrxC2 exist only under monomeric forms but with variable oxidation states. Except for the GSSG-treated GrxC1, there is a minor form with a mass decrease of about $2 \mathrm{Da}$ consistent with the formation of an intramolecular disulfide. Another minor form, found in all samples except the GSNO-treated GrxC2, exhibited a mass increment of $610.5-611 \mathrm{Da}$, which corresponded to the presence of two glutathione adducts. However, the major species formed by these two treatments is a monomer with a mass increment of about 303.5 Da which is consistent with the presence of one glutathione adduct on $\mathrm{Cys}_{C}$ in addition to the $\mathrm{Cys}_{\mathrm{A}}-\mathrm{Cys}_{\mathrm{B}}$ disulfide bond. Accordingly, the major species detected in protein variants mutated for $\mathrm{Cys}_{C}$ (Grx C1 C88S and GrxC2 C80S) is the one with an intramolecular disulfide (decrease of 2 Da upon GSSG or GSNO treatments). The glutathionylated forms detected by mass spectrometry were visible on gels (see Figures 3E,F).

The in vitro observation of intermolecular disulfide bonds in $\mathrm{GrxC} 1$ and $\mathrm{C} 2$, involving $\mathrm{Cys}_{C}$, raised the question of their possible reduction. Thus, we tested the ability of DTT but also of a physiological GSH reducing system composed of NADPH, $\mathrm{GR}$, and GSH to reduce this intermolecular disulfide, the formation of which was initiated by a $2 \mathrm{~h} \mathrm{H}_{2} \mathrm{O}_{2}$ treatment. As shown in Figure 4, both DTT and GSH are able to reduce GrxC1 and GrxC2 dimers to a similar extent. Whether minute amounts of reduced monomeric Grx initially present or formed can catalytically increase the reduction of dimeric Grxs in the presence of GSH cannot be excluded. Overall, it indicates that the formation of these disulfide-bridged forms of $\mathrm{GrxC} 1$ and $\mathrm{GrxC} 2$ isoforms is reversible, notably by GSH, a relevant cellular physiological reductant.

\section{REDOX PROPERTIES OF CLASS I GLUTAREDOXINS}

As in other thiol-dependent oxidoreductases, the catalytic efficiency of class I Grxs should be at least partially governed by thermodynamic parameters as the redox midpoint potentials of dithiol-disulfide couples and by the reactivity of the catalytic cysteine which is dependent on its $\mathrm{p} K_{a}$. Hence, we determined the Cys $_{\mathrm{A}} \mathrm{p} K_{a}$ of the four Grx isoforms using a method relying on iodoacetamide which is an alkylating reagent reacting with thiolates but not thiols. The $\mathrm{pH}$-dependent inactivation of WT Grx by iodoacetamide was followed by comparing the activity of WT proteins in the HED assay, after pre-incubation of the protein in 


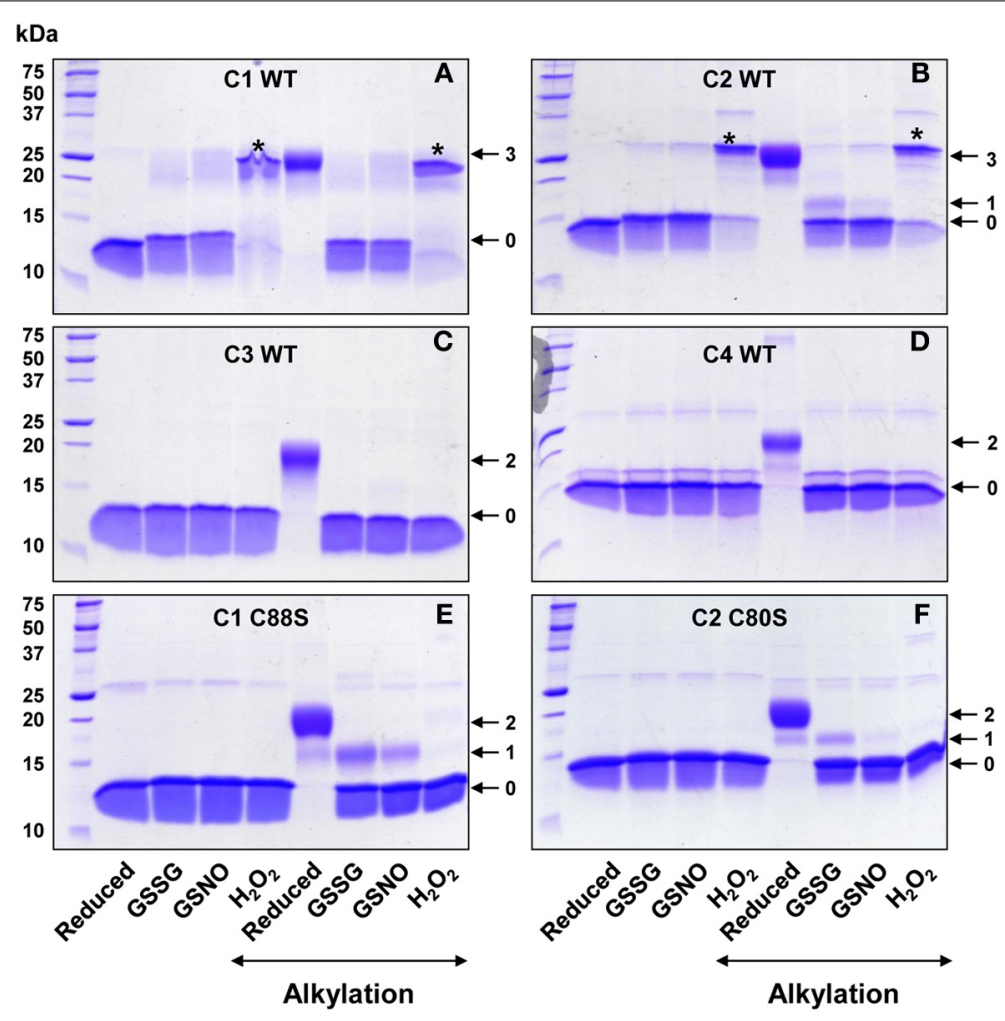

FIGURE 3 | Sensitivity of poplar Grxs to oxidative treatments. Pre-reduced Grxs were subjected to $2 \mathrm{~h}$ oxidative treatments with $\mathrm{H}_{2} \mathrm{O}_{2}$, GSSG, and GSNO as described in the "materials and methods" section. Prior to separation on SDS-PAGE gels in non-reducing conditions, GrxC1 WT (A), GrxC2 WT (B), GrxC3 WT (C), GrxC4 WT (D), GrxC1 C88S (E), and GrxC2 C80S (F) were alkylated or not with $2 \mathrm{kDa}$ mPEG maleimide. The shift observed following the alkylation of the free thiol groups is larger than expected (ca. $4 \mathrm{kDa}$ instead of $2 \mathrm{kDa}$ ), but this has been already observed previously (Chibani et al., 2011). The numbers on the right correspond to the number of alkylated thiols that remained free after reducing or oxidizing treatment of Grxs. The stars in panels $(\mathbf{A})$ and $(\mathbf{B})$ indicate the position of covalent dimer. different buffers ranging from $\mathrm{pH} 2.0$ to 7.0 in the presence or not of iodoacetamide. From these titration curves, we obtained $\mathrm{p} K_{a}$ values of $5.3 \pm 0.1$ for GrxC1, $5.0 \pm 0.1$ for GrxC2, $4.6 \pm 0.1$ for GrxC3, and $4.6 \pm 0.1$ for GrxC4 (Figure 5). These results indicate first that at physiological $\mathrm{pH}$, the thiolate ion will be the dominant species for all Grxs. Although the differences are not huge, it also confirmed the existence of two subgroups, GrxC1 and $\mathrm{C} 2$ having higher $\mathrm{p} K_{a}$ compared to GrxC3 and $\mathrm{C} 4$.

Two disulfide bond forms may be catalytically relevant for all these Grxs, the one with a glutathionylated $\mathrm{Cys}_{\mathrm{A}}$ and the one with a $\mathrm{Cys}_{\mathrm{A}}-\mathrm{Cys}_{\mathrm{B}}$ intramolecular disulfide bond. For measuring the midpoint redox potentials $\left(E_{m}\right)$ of each of these disulfide/dithiol couples, we used different protein variants and two distinct oxidative treatments in order to discriminate between these two oxidized forms. To obtain proteins containing an intramolecular disulfide bond, reduced Grxs possessing only the two active site cysteines (GrxC1 C88S, GrxC2 C80S, GrxC3 WT, and GrxC4 WT) have been treated with $\mathrm{H}_{2} \mathrm{O}_{2}$ before desalting on G25 columns. On the other hand, glutathionylated proteins have been obtained by treating reduced proteins in which only Cys $_{\mathrm{A}}$ was remaining (GrxC1 C34/88S, GrxC2 C26/80S, GrxC3 C40S, and GrxC4 C30S) with GSNO before desalting. The $E_{m}$ values determined at $\mathrm{pH} 7.0$ for the intramolecular disulfide range from $-233 \mathrm{mV}$ for GrxC3 to $-263 \mathrm{mV}$ to GrxC1 (Figure 6). On the other hand, only little variations were visible when measuring $E_{m}$ values for the glutathione adduct formed on the catalytic cysteine. They varied from $-242 \mathrm{mV}$ for GrxC3 to $-254 \mathrm{mV}$ for GrxC1. Overall, the intramolecular active site disulfide bond and glutathionylated forms of GrxC2 and GrxC4 present similar $E_{m}$ values. A $10 \mathrm{mV}$ difference was measured between both forms for $\mathrm{GrxC} 1$ and $\mathrm{C} 3$, the more electronegative redox potential being the active site disulfide in $\mathrm{GrxCl}$ and the glutathione adduct in GrxC3. However, the differences are small and should not explain the observed differences in catalytic efficiencies. In reducing cellular environments, all these oxidation forms should be compatible with glutathione reduction. Considering their possible targeting to secretory pathways, this may not be the case for Grx3 and C4 if they are in the endoplasmic reticulum or in the apoplasm.

\section{DISCUSSION}

DUPLICATION AND NEO-FUNCTIONALIZATION INTO CLASS I GLUTAREDOXINS

Among all living organisms, higher plants have the highest number of genes coding for Grxs. In addition to specific isoforms such as those belonging to the class III, land plants also possess 


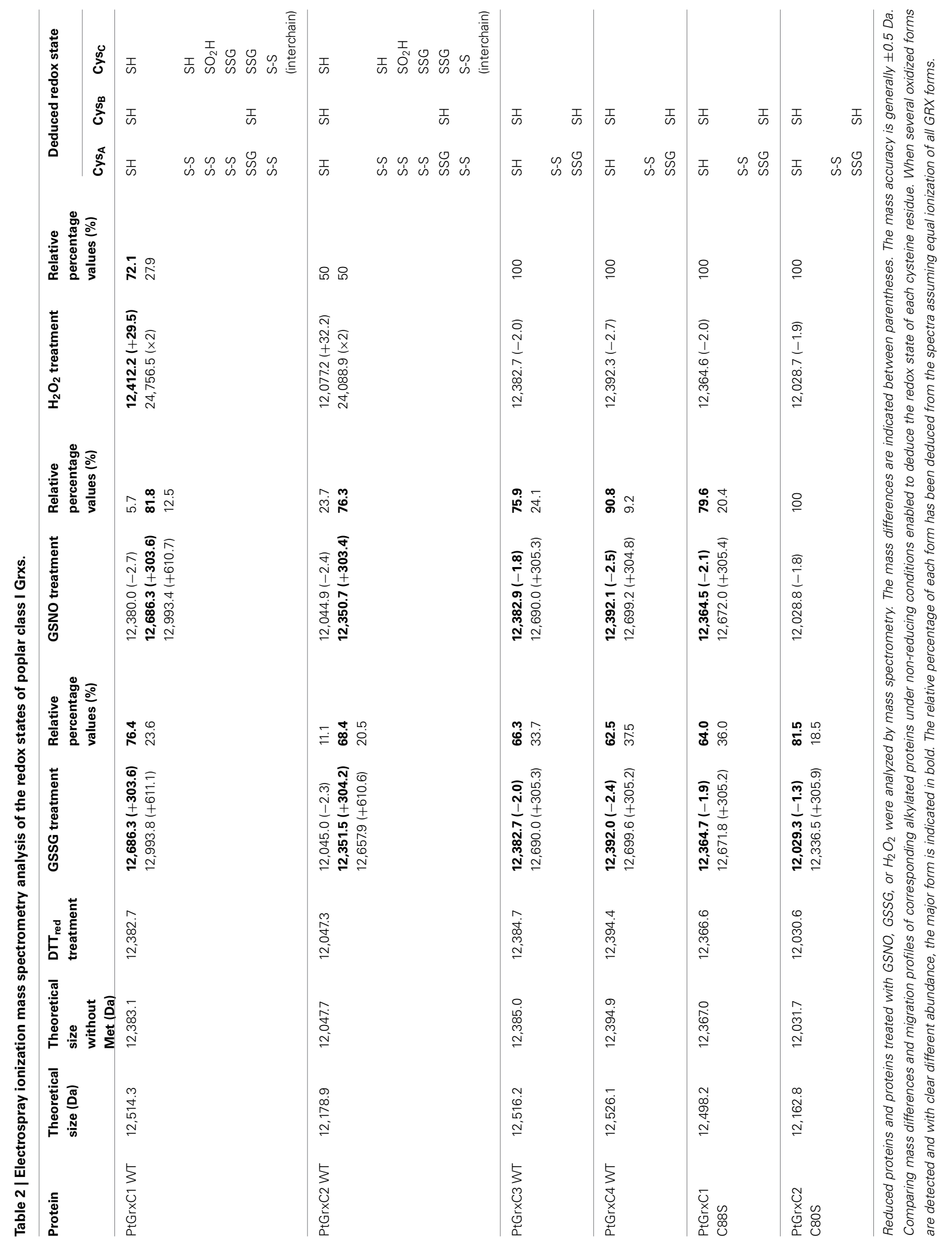




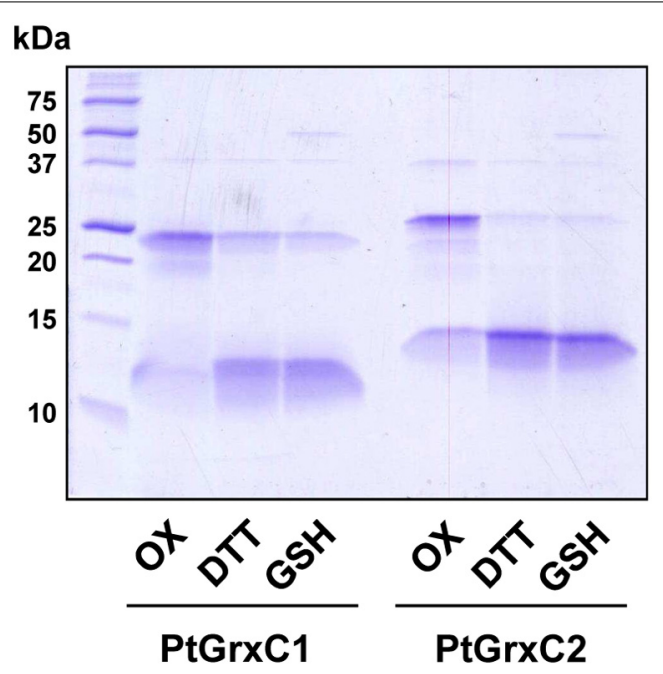

FIGURE 4 | GSH-dependent reduction of the intermolecular disulfide in poplar GrxC1 and GrxC2. The ability of GSH to reduce poplar disulfide-bridged dimer $\mathrm{GrxC} 1$ and disulfide-bridged dimer $\mathrm{GrxC2}$ generated by a $2 \mathrm{~h} \mathrm{H}_{2} \mathrm{O}_{2}$ treatment was evaluated in the presence of $1 \mathrm{mM}$ DTT and $1 \mathrm{mM}$ GSH coupled to NADPH and GR. The lanes Ox represent the initial oxidized proteins. Proteins were separated on SDS-PAGE gels under non-reducing conditions.
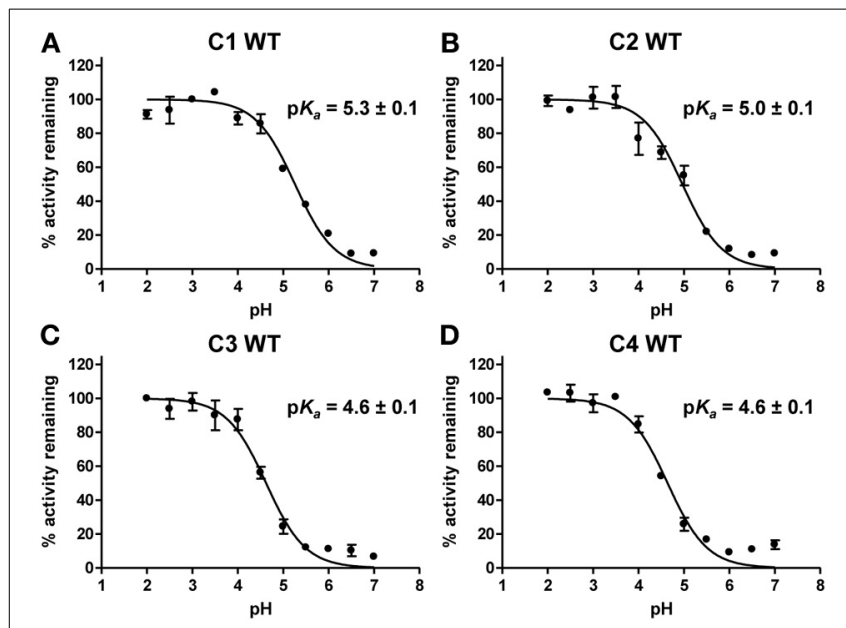

FIGURE 5 | $\mathrm{p} K_{a}$ determination of the catalytic cysteine of poplar GrxC1, C2, C3, and C4. Reduced GrxC1 (A), GrxC2 (B), GrxC3 (C), and GrxC4 (D) WT proteins were incubated in different buffers ranging from $\mathrm{pH}$ 2.0 to 7.0 in presence or not of iodoacetamide (IAM) prior to measurement of their activity by using the HED assay. The percentages of remaining activity at each $\mathrm{pH}$ were determined by comparing the activity of the enzyme incubated with and without IAM and an adaptation of the Henderson-Hasselbach equation was used for $p K_{a}$ value determination. The data are represented as mean $\pm S D$ of three separate experiments.

an expanded class I, usually with 5-6 isoforms (Couturier et al., 2009a). From previous phylogenetic analyses, it was quite clear that, there are three Grx couples, GrxC1/C2, GrxC3/C4, and GrxC5/S12 that probably evolved by more or less recent duplication (Couturier et al., 2009a). Interestingly, GrxC1 and C2 proteins are cytoplasmic proteins (Riondet et al., 2012), GrxC3

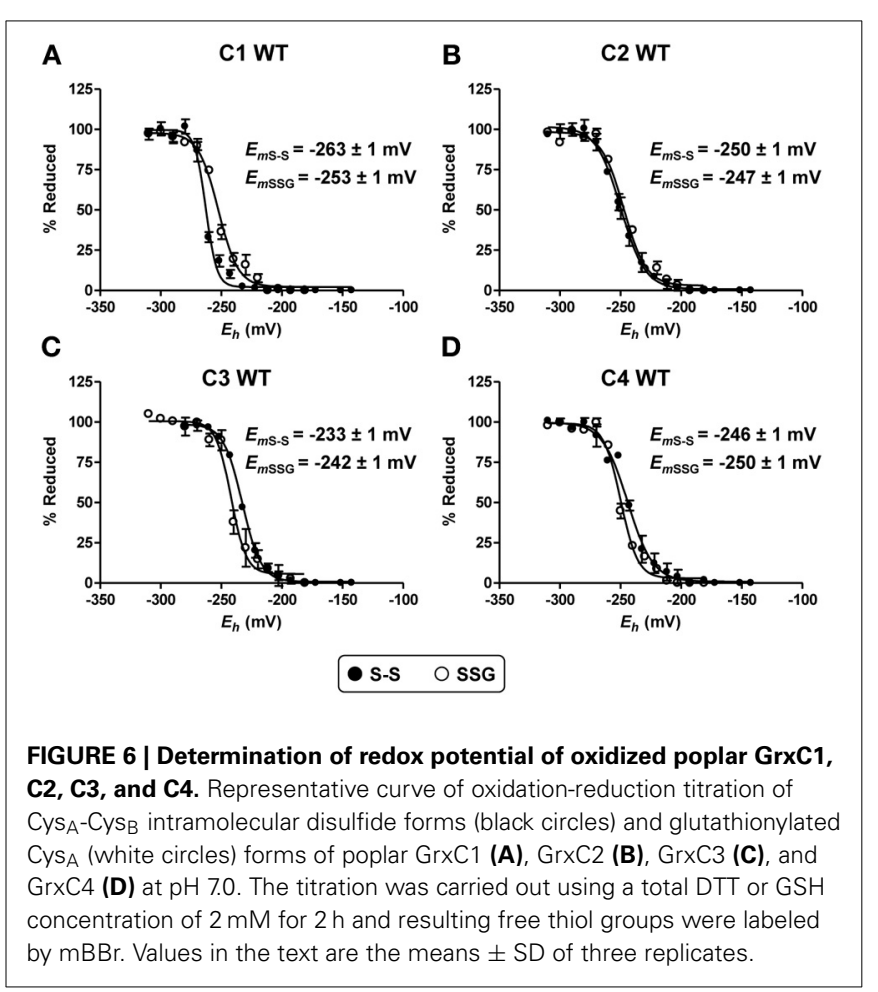

and C4 have N-terminal extensions predicted to target the proteins to secretory pathways but the final destination is not known, and GrxC5 and S12 are chloroplastic (Couturier et al., 2009b, 2011). The case of GrxC1 and C2 is interesting. Whereas GrxC2 prototypes are found in all land plants (mosses, lycophytes, monocots, dicots), GrxC1 is specifically found in dicots. A redundancy between both isoforms is suggested in A. thaliana by the observation that single knock-out mutants in grxc1 or grxc2 are aphenotypic, whereas a double mutant is lethal at an early stage after pollinization (Riondet et al., 2012). Considering that GrxC1, but not GrxC2, is able to bind an Fe-S cluster (at least in vitro), this would rather suggest that the essential nature of these two paralogs is linked to their reductase activity. These results point to the need of investigating at the genetic level a possible redundancy among the GrxC3/C4 and GrxC5/12 couples. This study primarily focused on the biochemical properties of dithiol class I Grxs since a detailed structure-function analysis was previously achieved on the two other prototypes of class I Grxs, the poplar monothiol GrxS12 and the Arabidopsis dithiol GrxC5 (Couturier et al., 2009b, 2011). GrxC5 isoforms are only found in species of the Brassicaceae family. We have demonstrated in particular that, although being very similar in sequence due to their recent duplication, a single substitution in the active site sequence (from WCSYS in GrxS12 to WCSYC in GrxC5) explains the capacity of GrxC5, but not GrxS12, to bind an $\left[\mathrm{Fe}_{2}-\mathrm{S}_{2}\right]$ cluster into a dimer. Among other class I Grxs, only GrxC1 can also exist under a holoform binding an Fe-S cluster (Rouhier et al., 2007). In this case, the mutational analysis showed that the presence of the glycine in the CGYC active site instead of a proline in other Grxs (CPFC in GrxC2 or CPYC in GrxC3 and C4) was sufficient to explain this property. Overall, this indicates that subtle sequence differences 
affecting key residues may provide different properties to otherwise quite close proteins. This prompted us to further analyze the various glutaredoxins of this class as $\mathrm{GrxC} 2$ and $\mathrm{GrxC} 3$ have been only poorly studied so far.

\section{TWO SUBGROUPS IN DITHIOL GrXS EXHIBIT DISTINCT KINETIC PROPERTIES}

Considering the divergence observed in the amino acid sequences, the first aim of this work was to perform a thorough comparative analysis of the biochemical properties of GrxC1, GrxC2, GrxC3, and GrxC4 from poplar. All these proteins exhibit deglutathionylation or reductase activities using classical HED and DHA assays with catalytic efficiencies $\left(10^{5} \mathrm{M}^{-1} \mathrm{~s}^{-1}\right.$ for HED and $10^{3}-10^{4} \mathrm{M}^{-1} \mathrm{~s}^{-1}$ for DHA) in the same range as those previously reported for plant and non-plant class I Grxs (Table 3). Two subgroups can however be distinguished, GrxC1 and GrxC2 appeared to be less efficient catalysts than GrxC3 and GrxC4. The difference is particularly clear when considering the turnover numbers $\left(\mathrm{k}_{\mathrm{cat}}\right)$ and the $\mathrm{k}_{\mathrm{cat}} / K_{\mathrm{DHA}}$ and $\mathrm{k}_{\mathrm{cat}} / K_{\mathrm{GSH}}$. This is not visible with $\mathrm{k}_{\mathrm{cat}} / K_{\beta}-M E-S G$ because the differences in the turnover number are compensated in this case by differences in apparent affinity in favor of GrxC1 and C2. An observation that may be interesting to understand these differences is that GrxC1/C2 and GrxC3/C4 should have quite different charge distribution at the protein surface that could account for substrate recognition and thus for the observed differences in catalytic efficiency. Indeed, their behavior on ion exchange chromatography indicates that GrxC1 and GrxC2 should be more basic having a pI close or higher than 8, whereas GrxC3 and GrxC4 should be more acidic having a pI value below 8 (theoretical pI estimations are indeed comprised between 5 and 6).

In order to further explain the differences between poplar class I Grxs, we determined the $\mathrm{p} K_{a}$ value of their catalytic cysteine, as it was proposed that reactivity of human and Chlamydomonas class I Grxs is partially related to the $\mathrm{p} K_{a}$ of $\mathrm{N}$-terminal active site cysteine (Gallogly et al., 2008; Gao et al., 2010). The values obtained for poplar Grxs are globally close to those reported for other plant and non-plant class I Grxs (Table 3), including the value of 3.9 determined for poplar GrxS12 using the same procedure (Zaffagnini et al., 2012a; Roos et al., 2013). Interestingly, a difference of ca 0.5 unit is visible between GrxC1/C2 ( $\mathrm{p} K_{a}$ around 5) and $\mathrm{GrxC} 3 / \mathrm{C} 4\left(\mathrm{p} K_{a}\right.$ around 4.6). Such differences between paralogs have been described already since C. reinhardtii Grx1, S. cerevisiae Grx2, and human Grx1 exhibit higher turnover numbers often translated into higher catalytic efficiencies compared to C. reinhardtii Grx2, S. cerevisiae Grx1, and human Grx2, respectively (Table 3 ). For the human and $C$. reinhardtii enzymes, it is also associated to a difference in $\mathrm{p} K_{a}$ values of $c a 1$ unit, the enzymes with the lowest $\mathrm{p} K_{a}$ being the most efficient. According to the Brønsted theory, in a thiol-disulfide exchange reaction, each unit decrease in the $\mathrm{p} K_{a}$ value of the leaving group is responsible for a 4 fold increase in the second-order rate constant (Szajewski and Whitesides, 1980). This could fit with the conclusion that differences in $\mathrm{p} K_{a}$ values between class I poplar Grx isoforms are at least partially responsible for the differences in catalytic efficiencies observed. On the other hand, only small differences were observed for the oxidation-reduction midpoint potentials of both the glutathione adduct or the intramolecular disulfide and they are not discriminating GrxC1/C2 vs. GrxC3/C4. The measured values (from -230 to $-260 \mathrm{mV}$ ) are in the same range than those reported for E. coli Grxl $(-233 \mathrm{mV})$ and human Grxl and 2 $(-232$ and $-221 \mathrm{mV}$ ) (Table 3) (Aslund et al., 1997; Sagemark et al., 2007).

\section{THE ROLE OF THE RECYCLING Cys B $_{\mathrm{B}}$ MONOTHIOL OR DITHIOL REACTION MECHANISM?}

Interestingly, the differences in protein activities between $\mathrm{GrxC1}$ or $\mathrm{C} 2$ and GrxC3 or $\mathrm{C} 4$ are attenuated when $\mathrm{Cys}_{\mathrm{B}}$ of $\mathrm{GrxC1}$ or C2 is removed (see Figure 2). This has been reported also for Arabidopsis GrxC5, yeast Grx1, human Grx1 and Grx2, and pig Grx (Yang and Wells, 1991; Yang et al., 1998; Johansson et al., 2004; Gallogly et al., 2008; Discola et al., 2009; Couturier et al., 2011). In clear contrast, mutating this cysteine in yeast Grx2, E. coli Grx1 and Grx3 led to a decrease of Grx activity (Bushweller et al., 1992; Nordstrand et al., 1999; Peltoniemi et al., 2006; Discola et al., 2009; Saaranen et al., 2009). For E. coli Grxl and by extension for Grxs for which mutation of this cysteine decreased their activity, this decrease was attributed to a change in glutathione recognition, binding, or affinity, because $\mathrm{Cys}_{\mathrm{B}}$ would determine the glutathione specificity of the glutathionylated Grx reduction step (Saaranen et al., 2009). For GrxC1 and GrxC2 and other eukaryote Grxs mentioned above in which the mutation of this cysteine increased their activity, the data indicates that the presence of $\mathrm{Cys}_{B}$ slows down the reaction for some reason. It may eventually modify the $\mathrm{p} K_{a}$ of $\mathrm{Cys}_{\mathrm{A}}$. This has been experimentally confirmed for a pig Grx, for which the $\mathrm{p} K_{a}$ of the catalytic Cys 22 is 3.8 in a WT protein but 4.9 and 5.9 in $\mathrm{C} 25 \mathrm{~S}$ and $\mathrm{C} 25 \mathrm{~A}$ variants (Yang and Wells, 1991). Another possibility is that a Cys ${ }_{A}-C_{B}$ intramolecular disulfide is formed during activity measurements either because it constitutes a regular step of the reaction cycle in some situations, especially for dithiol substrates or because it is a side reaction reflecting the competition between $\mathrm{Cys}_{\mathrm{B}}$ and $\mathrm{GSH}$ for the reduction of the glutathionylated Cys (Gallogly $_{\mathrm{A}}$ et al., 2009). Then, the reduction of this $\mathrm{Cys}_{\mathrm{A}}-\mathrm{Cys}_{\mathrm{B}}$ intramolecular disulfide intermediate would require two molecules of GSH vs. one molecule of GSH for the glutathionylated form and add two steps in the reaction mechanism. Thus, it seems unfavorable in terms of efficiency and energetic cost.

We have previously analyzed possible variations in the redox state of the dithiol AtGrxC5 (WCSYC active site) in response to 15 min treatments with GSSG or oxidized DTT by measuring the tryptophan intrinsic fluorescence of the protein, since the oxidation of the catalytic cysteine quenched the fluorescence of the adjacent tryptophan (Couturier et al., 2011). It was concluded that the dithiol AtGrxC5 cannot form a $\mathrm{Cys}_{\mathrm{A}}-\mathrm{Cys}_{\mathrm{B}}$ intramolecular disulfide first because only the GSSG treatment led to the formation of a glutathionylated protein and to fluorescence quenching and second because the WT protein was crystallized with a glutathionylated adduct. However, at that time, we did use neither $\mathrm{H}_{2} \mathrm{O}_{2}$ and GSNO treatments, nor prolonged incubation, nor alkylation assays. In fact, reconsidering the results of mass spectrometry showing, in addition to the peaks with one or two glutathione adducts, a peak with a decrease of $2 \mathrm{Da}$ between the reduced protein and the GSSG-treated protein, it is very likely 


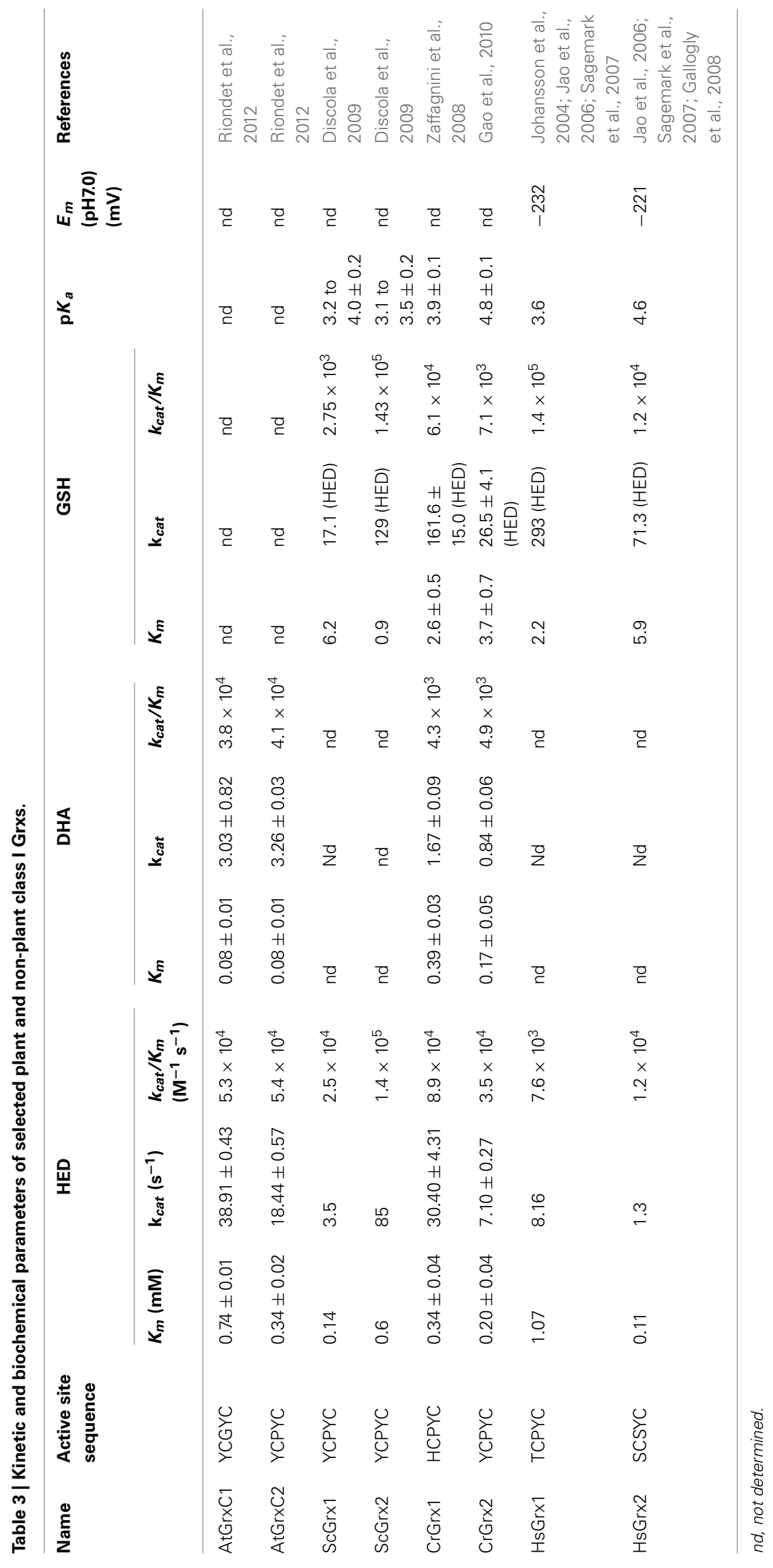


that a small fraction of the protein contained an intramolecular disulfide formed between active site cysteines (Couturier et al., 2011). Here, we have clearly observed that a prolonged oxidative treatment of reduced class I Grxs with $\mathrm{H}_{2} \mathrm{O}_{2}$, GSSG, or GSNO led preferentially to the formation of an intramolecular disulfide bond between $\mathrm{Cys}_{\mathrm{A}}$ and $\mathrm{Cys}_{\mathrm{B}}$. For the $\mathrm{H}_{2} \mathrm{O}_{2}$ treatment, it likely goes first through the formation of a sulfenic acid on $\mathrm{Cys}_{\mathrm{A}}$ before $\mathrm{Cys}_{\mathrm{B}}$ performs a nucleophilic attack on it. For the GSSG or GSNO treatments, it is very likely that a nucleophilic attack of $\mathrm{Cys}_{\mathrm{A}}$ onto these oxidized glutathione species leads to the formation of a glutathione adduct on $\mathrm{Cys}_{A}$ which is then slowly reduced by a $\mathrm{Cys}_{B}$ nucleophilic attack leading to the formation of the intramolecular disulfide and to the concomitant release of a GSH molecule.

It is worth mentioning here that these in vitro experiments have been done in particular with the aim of exploring all possible post-translational modifications but the conditions are not exactly physiological first because the concentrations of the oxidants may not be realistic and more importantly because this is done in the absence of GSH, which is normally found at high amounts and is highly reduced in most cell compartments. The presence of GSH would certainly prevent to a large extent the formation of these intramolecular disulfides because it may preferentially reduce either the sulfenic acids or the glutathione adducts over the $\mathrm{Cys}_{\mathrm{B}}$ recycling cysteine. This is certainly the case for GrxC3 and GrxC4 since mutating CysB has no effect on the activity. The formation of an intramolecular disulfide in these proteins could thus constitute uniquely a side reaction, protecting the cysteine or inactivating the proteins only under specific conditions. On the contrary, if we consider that the increase of protein activity observed for CysB mutated GrxC1 and GrxC2 in HED and DHA assays in the presence of a fully reduced GSH pool reflects the formation of this intramolecular disulfide, it may be physiologically relevant for GrxC1 and GrxC2. This is interesting in several respects. First, while the current dogma is that the preferential substrates of Grxs are glutathionylated proteins, recent studies performed with mammalian Grx2 indicated that they can reduce dithiol substrates, e.g., proteins with intra- or intermolecular disulfide (Hanschmann et al., 2010; Schutte et al., 2013). In this case, the Grxs should employ a reaction mechanism similar to the one used by Trxs involving both $\mathrm{Cys}_{\mathrm{A}}$ and $\mathrm{Cys}_{\mathrm{B}}$. Second, based on the observation that some Grxs can be reduced by thioredoxin reductases (Johansson et al., 2004; Zaffagnini et al., 2008; Couturier et al., 2013), another possibility could be that in specific physiological situations or sub-cellular compartments where glutathione is depleted, absent or oxidized, the formation of an intramolecular disulfide may be determinant either to favor the use of an alternative reducing system as the one constituted by NADPH and thioredoxin reductase or to simply constitute a protective mechanism, preventing the irreversible overoxidation of this residue and thus protein inactivation.

In conclusion, the role of $\mathrm{Cys}_{\mathrm{B}}$ in dithiol Grxs is yet uncertain and may depend on the Grx isoform as this residue influences differentially the protein activity. The fact that the formation of an intramolecular disulfide bond between $\mathrm{Cys}_{\mathrm{A}}$ and $\mathrm{Cys}_{\mathrm{B}}$ is possible in all cases lets open the possibility to use both monothiol and dithiol catalytic mechanisms, contrary to Grxs possessing only one cysteine residue.

\section{THE ROLE OF THE SEMI-CONSERVED Cys ${ }_{C}$ IN GrxC1 AND GrxC2 ISOFORMS}

The $\mathrm{Cys}_{C}$ cysteine residue, present in the IGGCD motif (at position 88 and 80 in GrxC1 and C2, respectively), is found in several other class I Grxs, including plant GrxC5 and S12 as well as in human Grx1 and E. coli Grx3. Moreover, it is also present in many class II Grxs. It was shown for instance that a $\mathrm{Cys}_{\mathrm{A}}-\mathrm{Cys} C$ intramolecular disulfide bond can be formed in S. cerevisiae Grx5 and C. reinhardtii Grx3 (Tamarit et al., 2003; Zaffagnini et al., 2008), suggesting that $\mathrm{Cys}_{C}$ can serve as a recycling cysteine in the absence of Cysi. Reduced glutathione does not seem to be an efficient reductant for this intramolecular disulfide bond and in the case of $\mathrm{CrGrx3}$, it is reduced by a ferredoxin-thioredoxin reductase system (Zaffagnini et al., 2008). Although GrxS12 has a monothiol WCSYS active site sequence, a Cys $_{A}-\mathrm{Cys}_{C}$ intramolecular disulfide has not been observed whatever the oxidizing conditions tested. Moreover, in the crystal structure of a glutathionylated GrxS12, the sulfur atom of Cys87 is 9.6 away from the Cys27-SG adduct (Couturier et al., 2009b). Hence, this cysteine may not serve as a recycling cysteine in class I Grxs and does not a priori participate to the reaction mechanism. Consistently, its mutation in GrxC1 and C2 did not influence protein activity, as previously observed for poplar GrxS12, Arabidopsis GrxC5, or E. coli Grx3 (Nordstrand et al., 1999; Couturier et al., 2009b, 2011).

However, considering its position very close to the active site residues and to residues involved in GSH binding an effect on activity could have been observed. Indeed, it is positioned between the so-called GG kink which is important in determining the backbone geometry of the following amino acids, and a Thr or an Asp which forms hydrogen bonds with GSH (see Figure 1) (Couturier et al., 2009b; Li et al., 2010). In the GrxS12 structure, the backbone amino group of $\mathrm{Cys}_{C}$ also directly forms a hydrogen bond with the glutamyl group of GSH (Couturier et al., 2009b), but the substitution of the cysteine into serine would not disrupt this interaction, preventing to definitely rule out a GSH stabilizing effect of this cysteine. On the other hand, it is probable that modifying the redox state of this cysteine might have more pronounced effects influencing for example GSH binding. It has been shown for example that, under non-reducing conditions, E. coli Grx3 exists under an expected monomeric form but also forms disulfide-bridged dimer or multimers via Cys $_{C}$ (Aslund et al., 1994, 1997). In addition, previously reported oxidative treatments of human Grx1, which contains three extra active site cysteines including $\mathrm{Cys}_{C}$, led to the identification of several possible post-translational modifications, i.e., intramolecular disulfide, disulfide-bonded dimers and oligomers, glutathione adducts, nitrosylation, some of them inhibiting its activity (Hashemy et al., 2007). Depending on the conditions, ${ }^{\text {Cys }} C$ was found to be either nitrosylated or glutathionylated or involved into a disulfide. Similarly, we have observed redox changes of GrxC1 and $\mathrm{C} 2$ upon treatment with oxidants that could further provide clues about the possible function of this cysteine, if any. In the presence of $\mathrm{H}_{2} \mathrm{O}_{2}$, this cysteine was found to be either overoxidized or involved in disulfide-bridged homodimers. 
Since this dimerization only occurs in presence of $\mathrm{H}_{2} \mathrm{O}_{2}$, the formation of an intermediate sulfenic acid is likely required before intermolecular disulfide bond formation which is consistent with the presence of a small part of overoxidized proteins. Interestingly, GSH can efficiently reduce these disulfide-bridged dimers, making this reaction physiologically reversible. In the presence of GSSG or GSNO, Cys $_{C}$ is also prone to oxidative modification, but in the form of a glutathione adduct. Although we cannot test the impact of these modifications on Grx activity (we cannot selectively modify $\mathrm{Cys}_{C}$ without also oxidizing $\mathrm{Cys}_{\mathrm{A}}$ and $\mathrm{Cys}_{\mathrm{B}}$ ), we can speculate that the different reversible oxidation forms of $\mathrm{Cys}_{C}$ observed here might represent regulatory or signaling intermediates transiently modifying the functions of these Grxs by affecting for example the nature or the time-course reduction of the target proteins.

\section{ACKNOWLEDGMENTS}

This work was supported by the French National Research Agency through the Laboratory of Excellence ARBRE (ANR12-LABXARBRE-01). The authors would like to thank Bruno Dastillung and Arnaud Hecker for their help with some experiments.

\section{SUPPLEMENTARY MATERIAL}

The Supplementary Material for this article can be found online at: http://www.frontiersin.org/journal/10.3389/fpls.2013. 00518/abstract

Figure S1 | Electrospray ionization mass spectrometry analysis of PtGrxC1 WT. Spectra of whole protein were determined for reduced protein before (A) and after treatment with GSSG (B), GSNO (C), or $\mathrm{H}_{2} \mathrm{O}_{2}$ (D) as described in the Methods section.

Figure S2 | Electrospray ionization mass spectrometry analysis of PtGrxC2 WT. Spectra of whole protein were determined for reduced protein before (A) and after treatment with GSSG (B), GSNO (C), or $\mathrm{H}_{2} \mathrm{O}_{2}$ (D) as described in the Methods section.

Figure S3 | Electrospray ionization mass spectrometry analysis of PtGrxC3 WT. Spectra of whole protein were determined for reduced protein before (A) and after treatment with GSSG (B), GSNO (C), or $\mathrm{H}_{2} \mathrm{O}_{2}$ (D) as described in the Methods section.

Figure S4 | Electrospray ionization mass spectrometry analysis of PtGrxC4 WT. Spectra of whole protein were determined for reduced protein before (A) and after treatment with GSSG (B), GSNO (C), or $\mathrm{H}_{2} \mathrm{O}_{2}$ (D) as described in the Methods section.

Figure S5 | Electrospray ionization mass spectrometry analysis of PtGrxC1 C88S. Spectra of whole protein were determined for reduced protein before (A) and after treatment with GSSG (B), GSNO (C), or $\mathrm{H}_{2} \mathrm{O}_{2}$ (D) as described in the Methods section.

Figure S6 | Electrospray ionization mass spectrometry analysis of PtGrxC2 C80S. Spectra of whole protein were determined for reduced protein before (A) and after treatment with GSSG (B), GSNO (C), or $\mathrm{H}_{2} \mathrm{O}_{2}$ (D) as described in the Methods section.

Table S1 | Primers used for site-directed mutagenesis.

\section{REFERENCES}

Alves, R., Vilaprinyo, E., Sorribas, A., and Herrero, E. (2009). Evolution based on domain combinations: the case of glutaredoxins. BMC Evol. Biol. 9:66. doi: 10.1186/1471-2148-9-66

Aslund, F., Berndt, K. D., and Holmgren, A. (1997). Redox potentials of glutaredoxins and other thiol-disulfide oxidoreductases of the thioredoxin superfamily determined by direct protein-protein redox equilibria. J. Biol. Chem. 272, 30780-30786. doi: 10.1074/jbc.272.49.30780

Aslund, F., Ehn, B., Miranda-Vizuete, A., Pueyo, C., and Holmgren, A. (1994). Two additional glutaredoxins exist in Escherichia coli: glutaredoxin 3 is a hydrogen donor for ribonucleotide reductase in a thioredoxin/glutaredoxin 1 double mutant. Proc. Natl. Acad. Sci. U.S.A. 91, 9813-9817. doi: 10.1073/pnas.91.21.9813

Bandyopadhyay, S., Gama, F., Molina-Navarro, M. M., Gualberto, J. M., Claxton, R., Naik, S. G., et al. (2008). Chloroplast monothiol glutaredoxins as scaffold proteins for the assembly and delivery of [2Fe-2S] clusters. EMBO J. 27, 1122-1133. doi: 10.1038/emboj.2008.50

Bedhomme, M., Adamo, M., Marchand, C. H., Couturier, J., Rouhier, N., Lemaire, S. D., et al. (2012). Glutathionylation of cytosolic glyceraldehyde-3-phosphate dehydrogenase from the model plant Arabidopsis thaliana is reversed by both glutaredoxins and thioredoxins in vitro. Biochem. J. 445, 337-347. doi: 10.1042/BJ20120505

Bushweller, J. H., Aslund, F., Wuthrich, K., and Holmgren, A. (1992). Structural and functional characterization of the mutant Escherichia coli glutaredoxin (C14-S) and its mixed disulfide with glutathione. Biochemistry 31, 9288-9293. doi: 10.1021/bi00153a023

Chibani, K., Tarrago, L., Gualberto, J. M., Wingsle, G., Rey, P., Jacquot, J. P., et al. (2012). Atypical thioredoxins in poplar: the glutathione-dependent thioredoxin-like 2.1 supports the activity of target enzymes possessing a single redox active cysteine. Plant Physiol. 159, 592-605. doi: 10.1104/pp.112.197723

Chibani, K., Tarrago, L., Schurmann, P., Jacquot, J. P., and Rouhier, N. (2011). Biochemical properties of poplar thioredoxin z. FEBS Lett. 585, 1077-1081. doi: 10.1016/j.febslet.2011.03.006

Couturier, J., Didierjean, C., Jacquot, J. P., and Rouhier, N. (2010). Engineered mutated glutaredoxins mimicking peculiar plant class III glutaredoxins bind iron-sulfur centers and possess reductase activity. Biochem. Biophys. Res. Commun. 403, 435-441. doi: 10.1016/j.bbrc.2010.11.050

Couturier, J., Jacquot, J. P., and Rouhier, N. (2009a). Evolution and diversity of glutaredoxins in photosynthetic organisms. Cell. Mol. Life Sci. 66, 2539-2557. doi: 10.1007/s00018-009-0054-y

Couturier, J., Koh, C. S., Zaffagnini, M., Winger, A. M., Gualberto, J. M., Corbier, C., et al. (2009b). Structure-function relationship of the chloroplastic glutaredoxin S12 with an atypical WCSYS active site. J. Biol. Chem. 284, 9299-9310. doi: 10.1074/jbc.M807998200

Couturier, J., Prosper, P., Winger, A. M., Hecker, A., Hirasawa, M., Knaff, D. B., et al. (2013). In the absence of thioredoxins, what are the reductants for peroxiredoxins in Thermotoga maritima? Antioxid. Redox Signal. 18, 1613-1622. doi: 10.1089/ars.2012.4739

Couturier, J., Stroher, E., Albetel, A. N., Roret, T., Muthuramalingam, M., Tarrago, L., et al. (2011). Arabidopsis chloroplastic glutaredoxin C5 as a model to explore molecular determinants for iron-sulfur cluster binding into glutaredoxins. J. Biol. Chem. 286, 27515-27527. doi: 10.1074/jbc.M111.228726

Dalle-Donne, I., Rossi, R., Colombo, G., Giustarini, D., and Milzani, A. (2009). Protein S-glutathionylation: a regulatory device from bacteria to humans. Trends Biochem. Sci. 34, 85-96. doi: 10.1016/j.tibs.2008.11.002

Discola, K. F., De Oliveira, M. A., Rosa Cussiol, J. R., Monteiro, G., Barcena, J. A., Porras, P., et al. (2009). Structural aspects of the distinct biochemical properties of glutaredoxin 1 and glutaredoxin 2 from Saccharomyces cerevisiae. J. Mol. Biol. 385, 889-901. doi: 10.1016/j.jmb.2008.10.055

Finkemeier, I., Goodman, M., Lamkemeyer, P., Kandlbinder, A., Sweetlove, L. J., and Dietz, K. J. (2005). The mitochondrial type II peroxiredoxin F is essential for redox homeostasis and root growth of Arabidopsis thaliana under stress. J. Biol. Chem. 280, 12168-12180. doi: 10.1074/jbc.M413189200

Gallogly, M. M., Starke, D. W., Leonberg, A. K., Ospina, S. M., and Mieyal, J. J. (2008). Kinetic and mechanistic characterization and versatile catalytic properties of mammalian glutaredoxin 2: implications for intracellular roles. Biochemistry 47, 11144-11157. doi: 10.1021/bi800966v

Gallogly, M. M., Starke, D. W., and Mieyal, J. J. (2009). Mechanistic and kinetic details of catalysis of thiol-disulfide exchange by glutaredoxins and 
potential mechanisms of regulation. Antioxid. Redox Signal. 11, 1059-1081. doi: 10.1089/ars.2008.2291

Gao, X. H., Zaffagnini, M., Bedhomme, M., Michelet, L., Cassier-Chauvat, C., Decottignies, P., et al. (2010). Biochemical characterization of glutaredoxins from Chlamydomonas reinhardtii: kinetics and specificity in deglutathionylation reactions. FEBS Lett. 584, 2242-2248. doi: 10.1016/j.febslet.2010. 04.034

Hanschmann, E. M., Lonn, M. E., Schutte, L. D., Funke, M., Godoy, J. R., Eitner, S., et al. (2010). Both thioredoxin 2 and glutaredoxin 2 contribute to the reduction of the mitochondrial 2-Cys peroxiredoxin Prx3. J. Biol. Chem. 285, 40699-40705. doi: 10.1074/jbc.M110.185827

Hashemy, S. I., Johansson, C., Berndt, C., Lillig, C. H., and Holmgren, A. (2007). Oxidation and S-nitrosylation of cysteines in human cytosolic and mitochondrial glutaredoxins: effects on structure and activity. J. Biol. Chem. 282, 14428-14436. doi: 10.1074/jbc.M700927200

Jao, S. C., English Ospina, S. M., Berdis, A. J., Starke, D. W., Post, C. B., and Mieyal, J. J. (2006). Computational and mutational analysis of human glutaredoxin (thioltransferase): probing the molecular basis of the low pKa of cysteine 22 and its role in catalysis. Biochemistry 45, 4785-4796. doi: 10.1021/bi0516327

Johansson, C., Lillig, C. H., and Holmgren, A. (2004). Human mitochondrial glutaredoxin reduces S-glutathionylated proteins with high affinity accepting electrons from either glutathione or thioredoxin reductase. J. Biol. Chem. 279, 7537-7543. doi: 10.1074/jbc.M312719200

Li, W. F., Yu, J., Ma, X. X., Teng, Y. B., Luo, M., Tang, Y. J., et al. (2010). Structural basis for the different activities of yeast Grx1 and Grx2. Biochim. Biophys. Acta 1804, 1542-1547. doi: 10.1016/j.bbapap.2010.04.010

Meyer, Y., Belin, C., Delorme-Hinoux, V., Reichheld, J. P., and Riondet, C. (2012). Thioredoxin and glutaredoxin systems in plants: molecular mechanisms, crosstalks, and functional significance. Antioxid. Redox Signal. 17, 1124-1160. doi: 10.1089/ars.2011.4327

Nordstrand, K., Aslund, F., Meunier, S., Holmgren, A., Otting, G., and Berndt, K. D. (1999). Direct NMR observation of the Cys-14 thiol proton of reduced Escherichia coli glutaredoxin-3 supports the presence of an active site thiol-thiolate hydrogen bond. FEBS Lett. 449, 196-200. doi: 10.1016/S00145793(99)00401-9

Peltoniemi, M. J., Karala, A. R., Jurvansuu, J. K., Kinnula, V. L., and Ruddock, L. W. (2006). Insights into deglutathionylation reactions. Different intermediates in the glutaredoxin and protein disulfide isomerase catalyzed reactions are defined by the gamma-linkage present in glutathione. J. Biol. Chem. 281, 33107-33114. doi: 10.1074/jbc.M605602200

Riondet, C., Desouris, J. P., Montoya, J. G., Chartier, Y., Meyer, Y., and Reichheld, J. P. (2012). A dicotyledon-specific glutaredoxin GRXC1 family with dimerdependent redox regulation is functionally redundant with GRXC2. Plant Cell Environ. 35, 360-373. doi: 10.1111/j.1365-3040.2011.02355.x

Rodriguez-Manzaneque, M. T., Ros, J., Cabiscol, E., Sorribas, A., and Herrero, E. (1999). Grx5 glutaredoxin plays a central role in protection against protein oxidative damage in Saccharomyces cerevisiae. Mol. Cell. Biol. 19, 8180-8190.

Roos, G., Foloppe, N., and Messens, J. (2013). Understanding the pK(a) of redox cysteines: the key role of hydrogen bonding. Antioxid. Redox Signal. 18, 94-127. doi: 10.1089/ars.2012.4521

Rouhier, N. (2010). Plant glutaredoxins: pivotal players in redox biology and iron-sulphur centre assembly. New Phytol. 186, 365-372. doi: 10.1111/j.14698137.2009.03146.x

Rouhier, N., Couturier, J., Johnson, M. K., and Jacquot, J. P. (2010). Glutaredoxins: roles in iron homeostasis. Trends Biochem. Sci. 35, 43-52. doi: 10.1016/j.tibs.2009.08.005

Rouhier, N., Gelhaye, E., and Jacquot, J. P. (2002). Exploring the active site of plant glutaredoxin by site-directed mutagenesis. FEBS Lett. 511, 145-149. doi: 10.1016/S0014-5793(01)03302-6

Rouhier, N., Gelhaye, E., Sautiere, P. E., Brun, A., Laurent, P., Tagu, D., et al. (2001). Isolation and characterization of a new peroxiredoxin from poplar sieve tubes that uses either glutaredoxin or thioredoxin as a proton donor. Plant Physiol. 127, 1299-1309. doi: 10.1104/pp.010586

Rouhier, N., Lemaire, S. D., and Jacquot, J. P. (2008). The role of glutathione in photosynthetic organisms: emerging functions for glutaredoxins and glutathionylation. Annu. Rev. Plant Biol. 59, 143-166. doi: 10.1146/annurev.arplant.59.032607.092811
Rouhier, N., Unno, H., Bandyopadhyay, S., Masip, L., Kim, S. K., Hirasawa, M., et al. (2007). Functional, structural, and spectroscopic characterization of a glutathione-ligated [2Fe-2S] cluster in poplar glutaredoxin C1. Proc. Natl. Acad. Sci. U.S.A. 104, 7379-7384. doi: 10.1073/pnas.0702268104

Saaranen, M. J., Salo, K. E., Latva-Ranta, M. K., Kinnula, V. L., and Ruddock, L. W. (2009). The C-terminal active site cysteine of Escherichia coli glutaredoxin 1 determines the glutathione specificity of the second step of peptide deglutathionylation. Antioxid. Redox Signal. 11, 1819-1828. doi: 10.1089/ars.2008.2387

Sagemark, J., Elgan, T. H., Burglin, T. R., Johansson, C., Holmgren, A., and Berndt, K. D. (2007). Redox properties and evolution of human glutaredoxins. Proteins 68, 879-892. doi: 10.1002/prot.21416

Schutte, L. D., Baumeister, S., Weis, B., Hudemann, C., Hanschmann, E. M., and Lillig, C. H. (2013). Identification of potential protein dithiol-disulfide substrates of mammalian Grx2. Biochim. Biophys. Acta 1830, 4999-5005. doi: 10.1016/j.bbagen.2013.07.009

Szajewski, R. P., and Whitesides, G. M. (1980). Rate constants and equilibrium constants for thiol-disulfide interchange reactions involving oxidized glutathione. J. Am. Chem. Soc. 102, 2011-2026. doi: 10.1021/ja00526a042

Tamarit, J., Belli, G., Cabiscol, E., Herrero, E., and Ros, J. (2003). Biochemical characterization of yeast mitochondrial Grx 5 monothiol glutaredoxin. J. Biol. Chem. 278, 25745-25751. doi: 10.1074/jbc.M303477200

Tarrago, L., Laugier, E., Zaffagnini, M., Marchand, C., Le Marechal, P., Rouhier, N., et al. (2009). Regeneration mechanisms of Arabidopsis thaliana methionine sulfoxide reductases B by glutaredoxins and thioredoxins. J. Biol. Chem. 284, 18963-18971. doi: 10.1074/jbc.M109.015487

Vieira Dos Santos, C., Laugier, E., Tarrago, L., Massot, V., Issakidis-Bourguet, E., Rouhier, N., et al. (2007). Specificity of thioredoxins and glutaredoxins as electron donors to two distinct classes of Arabidopsis plastidial methionine sulfoxide reductases B. FEBS Lett. 581, 4371-4376. doi: 10.1016/j.febslet.2007.07.081

Yang, Y., Jao, S., Nanduri, S., Starke, D. W., Mieyal, J. J., and Qin, J. (1998). Reactivity of the human thioltransferase (glutaredoxin) C7S, C25S, C78S, C82S mutant and NMR solution structure of its glutathionyl mixed disulfide intermediate reflect catalytic specificity. Biochemistry 37, 17145-17156. doi: 10.1021/bi9806504

Yang, Y. F., and Wells, W. W. (1991). Identification and characterization of the functional amino acids at the active center of pig liver thioltransferase by site-directed mutagenesis. J. Biol. Chem. 266, 12759-12765.

Zaffagnini, M., Bedhomme, M., Marchand, C. H., Couturier, J. R., Gao, X. H., Rouhier, N., et al. (2012a). Glutaredoxin s12: unique properties for redox signaling. Antioxid. Redox Signal. 16, 17-32. doi: 10.1089/ars.2011.3933

Zaffagnini, M., Bedhomme, M., Marchand, C. H., Morisse, S., Trost, P., and Lemaire, S. D. (2012b). Redox regulation in photosynthetic organisms: focus on glutathionylation. Antioxid. Redox Signal. 16, 567-586. doi: 10.1089/ars.2011.4255

Zaffagnini, M., Michelet, L., Massot, V., Trost, P., and Lemaire, S. D. (2008). Biochemical characterization of glutaredoxins from Chlamydomonas reinhardtii reveals the unique properties of a chloroplastic CGFS-type glutaredoxin. J. Biol. Chem. 283, 8868-8876. doi: 10.1074/jbc.M709567200

Conflict of Interest Statement: The authors declare that the research was conducted in the absence of any commercial or financial relationships that could be construed as a potential conflict of interest.

Received: 19 September 2013; accepted: 02 December 2013; published online: 18 December 2013.

Citation: Couturier J, Jacquot J-P and Rouhier N (2013) Toward a refined classification of class I dithiol glutaredoxins from poplar: biochemical basis for the definition of two subclasses. Front. Plant Sci. 4:518. doi: 10.3389/fpls.2013.00518

This article was submitted to Plant Physiology, a section of the journal Frontiers in Plant Science.

Copyright (c) 2013 Couturier, Jacquot and Rouhier. This is an open-access article distributed under the terms of the Creative Commons Attribution License (CC BY). The use, distribution or reproduction in other forums is permitted, provided the original author(s) or licensor are credited and that the original publication in this journal is cited, in accordance with accepted academic practice. No use, distribution or reproduction is permitted which does not comply with these terms. 